MATHEMATICS OF COMPUTATION

Volume 76, Number 258, April 2007, Pages 811-843

S 0025-5718(06)01939-9

Article electronically published on November 28, 2006

\title{
THE 192 SOLUTIONS OF THE HEUN EQUATION
}

\author{
ROBERT S. MAIER
}

\begin{abstract}
A machine-generated list of 192 local solutions of the Heun equation is given. They are analogous to Kummer's 24 solutions of the Gauss hypergeometric equation, since the two equations are canonical Fuchsian differential equations on the Riemann sphere with four and three singular points, respectively. Tabulation is facilitated by the identification of the automorphism group of the equation with $n$ singular points as the Coxeter group $\mathcal{D}_{n}$. Each of the 192 expressions is labeled by an element of $\mathcal{D}_{4}$. Of the 192, 24 are equivalent expressions for the local Heun function $\mathrm{Hl}$, and it is shown that the resulting order-24 group of transformations of $\mathrm{Hl}$ is isomorphic to the symmetric group $S_{4}$. The isomorphism encodes each transformation as a permutation of an abstract four-element set, not identical to the set of singular points.
\end{abstract}

\section{INTRODUCTION}

The theory of second-order linear differential equations on the Riemann sphere $\mathbb{P}^{1}(\mathbb{C}) \ni x$, with coefficients in the field of rational functions $\mathbb{C}(x)$, has been developed in both conceptual and algorithmic directions. In a modern interpretation, such equations correspond to flat meromorphic connections on two-dimensional vector bundles over $\mathbb{P}^{1}(\mathbb{C})$ [26]. The study of these equations and their solutions led to the classical theory of special functions [17.

The simplest, but still nontrivial case is the Fuchsian one, in which all singular points of the equation are regular. If there are $n$ regular singular points on $\mathbb{P}^{1}(\mathbb{C})$ $(n \geqslant 3)$, one of which is allowed to be $\infty$, the equation will be determined up to isomorphism by their locations, a parameter associated with each singular point (i.e., a difference of characteristic exponents), and $n-3$ accessory parameters, which have more a global than a local significance. The case $n=3$ is the simplest. By appropriate normalizations, any Fuchsian equation with three singular points can be reduced to the Gauss hypergeometric equation

$$
D_{x}^{2} u+\left[\frac{c}{x}+\frac{a+b-c+1}{x-1}\right] D_{x} u+\left[\frac{a b}{x(x-1)}\right] u=0,
$$

which has singular points $x=0,1, \infty$, and respective characteristic exponents $0,1-c ; 0,-a-b+c ; a, b$. The analysis of (1.1) led to much interesting nineteenthcentury mathematics [9]. Kummer's 24 local solutions of this equation, reproduced in Table11 below, are especially well known [1, 5, 6, 9, 17, 18, 28. Each is expressed in terms of the Gauss hypergeometric function ${ }_{2} F_{1}$. The 24 solutions split into

Received by the editor August 23, 2004 and, in revised form, February 7, 2006.

2000 Mathematics Subject Classification. Primary 33E30; Secondary 33-04, 34M15, 33C05, $20 \mathrm{~F} 55$.

The author was supported in part by NSF Grant No. PHY-0099484.

(C)2006 American Mathematical Society Reverts to public domain 28 years from publication 
6 sets of 4 formally distinct but equivalent expressions, each set defining one of the two Frobenius solutions in the neighborhood of a singular point.

The classical study of the hypergeometric equation was facilitated by its having no accessory parameters, and the situation when $n>3$ is significantly more complicated. It is known that any Fuchsian equation with $n=4$ singular points can be reduced to the Heun equation [19]. This is the equation

$$
D_{x}^{2} u+\left[\frac{\gamma}{x}+\frac{\delta}{x-1}+\frac{\epsilon}{x-a}\right] D_{x} u+\left[\frac{\alpha \beta x-q}{x(x-1)(x-a)}\right] u=0,
$$

which has singular points $x=0,1, a, \infty$, where $a \in \mathbb{C} \backslash\{0,1\}$ is a free parameter. The respective exponents are $0,1-\gamma ; 0,1-\delta ; 0,1-\epsilon ; \alpha, \beta$. It follows from Fuchs's relation on the exponents of Fuchsian equations that $\epsilon=\alpha+\beta-\gamma-\delta+1$. (If this condition does not hold, the singular point $x=\infty$ will not be regular.) The Heun equation includes an accessory parameter, namely the quantity $q \in \mathbb{C}$, which in many applications appears as a spectral parameter. This equation is under active study [15], in part because the band structure of its solutions is important to the theory of integrable nonlinear wave equations $[8,23$. Fuchsian equations with $n=5$ singular points are also being systematically studied 24 .

Kummer's family of 24 solutions of (1.1) has an analogue for each $n \geqslant 3$, namely a family of $2^{n-1} n$ ! local solutions, which splits into $2 n$ sets of $2^{n-2}(n-1)$ ! equivalent expressions, each set defining one of the two Frobenius solutions in the neighborhood of a singular point. (The $n$ ! factor comes from permuting the $n$ singular points, and the $2^{n-1}$ factor from negating exponent differences.) It is quite difficult to write down the full family of $2^{n-1} n$ ! solutions explicitly when $n>3$, especially if one uses hand computation. The $n=4$ case is a case in point. Just as each of Kummer's 24 solutions can be written in terms of the Gauss function ${ }_{2} F_{1}$, each of the $2^{3} \cdot 4 !=192$ solutions of the Heun equation can be written in terms of a canonical "local Heun function" $\mathrm{Hl}$. But no satisfactory list of these solutions has been published, until now. The stumbling block is the calculation, in each, of the value of the accessory parameter of $\mathrm{Hl}$. Heun listed 48 of the 192 in his 1889 paper [11, with the accessory parameter omitted in each. Applied mathematicians have noticed that apart from this, his list contains misprints, and cannot be used in practical applications [22, $\S 6.3]$. As an alternative the work of Snow [25] is frequently cited, since it gives 25 of the 192, in a slightly individualistic notation. Several solutions are given in the useful review of the late F. Arscott [19, Part A], which also discusses how they can be transformed into one another.

In this paper we determine the group structure of the set of transformations that can be applied to any normalized Fuchsian equation on $\mathbb{P}^{1}(\mathbb{C})$ with $n$ singular points, to generate alternative expressions for its family of local solutions. This automorphism group has order $2^{n-1} n$ ! and acts on the parameter space of the equation. As Theorem 4.3 states, it is isomorphic to the Coxeter group $\mathcal{D}_{n}[10$, the group of even-signed permutations of an $n$-set. This is an index- 2 normal subgroup of the hyperoctahedral Coxeter group $\mathcal{B}_{n}$, which is the group of signed permutations of an $n$-set. ( $\mathcal{B}_{n}$ itself is isomorphic to the wreath product $\mathbb{Z}_{2} \succ S_{n}$, where $S_{n}$ is the symmetric group.) A similar result was recently obtained by Oblezin [16], in an abstract framework. That the automorphism group is $\mathcal{D}_{n} \triangleleft \mathcal{B}_{n}=\left[\mathcal{D}_{n}: \mathbb{Z}_{2}\right]$ clarifies an observation of Dwork [5 that the "Kummer automorphism group", the order-24 automorphism group of the Gauss hypergeometric equation $(n=3)$, is isomorphic to the octahedral rotation group $S_{4}$, and has a natural $\mathbb{Z}_{2}$-extension to 
an order- 48 group. It is an accident that this order- 24 group is isomorphic to a symmetric group; there is no counterpart to the isomorphism $\mathcal{D}_{3} \cong S_{4}$ when $n>3$.

The identification of the automorphism group of the Heun equation, as $\mathcal{D}_{4}$, facilitates both hand and machine computations of the 192 solutions, including accessory parameter values. Only a few generating transformations need to be repeatedly applied. The solutions, indexed by $g \in \mathcal{D}_{4}$, are presented in Table 2 , It unfortunately follows from this table that nearly all the 48 solutions generated by Heun [11 contain at least one error. The errors are more serious than mere misprints.

Interestingly, the 24 solutions equivalent to $\mathrm{Hl}$ (including $\mathrm{Hl}$ itself) yield transformations of $H l$ which under composition, form an order-24 subgroup of $\mathcal{D}_{4}$ isomorphic to the Kummer automorphism group $\mathcal{D}_{3}$, and hence to $S_{4}$. The isomorphism to $S_{4}$ is given in Table 3. The possibility of encoding the 24 basic transformations of $\mathrm{Hl}$ as the permutations of an abstract four-element set, not to be identified with the set of four singular points, seems not to have been noticed before, though it is useful in hand computation.

The question of the global structure of the space of Fuchsian equations with $n$ singular points, up to isomorphism, is not addressed here. The space of three distinct points in $\mathbb{P}^{1}(\mathbb{C})$ is effectively a single point, since any two such configurations are related by a unique Möbius transformation; and the space of four distinct points in $\mathbb{P}^{1}(\mathbb{C})$ is effectively $\mathbb{C}$, which compactifies to $\mathbb{P}^{1}(\mathbb{C})$. The global structure of the compactified space of five-point configurations has only recently been determined [29]. Supplementing the singular points by exponent differences and accessory parameters makes global issues significantly more difficult to treat.

\section{BASIC FACTS}

Any second-order Fuchsian equation on $\mathbb{P}^{1}(\mathbb{C})$ with $n-1$ distinct finite singular points $d_{1}, \ldots, d_{n-1} \in \mathbb{C}$ can be written as $[17$ ]

$$
\begin{aligned}
T u \stackrel{\text { def }}{=} D_{x}^{2} u+\left[\sum_{i=1}^{n-1} \frac{1-\rho_{i}-\hat{\rho}_{i}}{x-d_{i}}\right] & D_{x} u \\
& +\left[\sum_{i=1}^{n-1} \frac{\rho_{i} \hat{\rho}_{i}}{\left(x-d_{i}\right)^{2}}+\frac{\Pi_{n-3}(x)}{\prod_{i=1}^{n-1}\left(x-d_{i}\right)}\right] u=0 .
\end{aligned}
$$

Here $\rho_{i}, \hat{\rho}_{i} \in \mathbb{C}$ are the characteristic exponents at the point $d_{i}$, which can be calculated by the method of Frobenius as the roots of an indicial equation. $\Pi_{n-3}$ is a polynomial of degree $n-3$, the leading coefficient of which enters into the indicial equation at the point $x=\infty$. The $2 n$ exponents, including the two exponents $\rho^{(\infty)}, \hat{\rho}^{(\infty)}$ at infinity, obey Fuchs's relation; their sum is $n-2$. The $n-3$ trailing coefficients of $\Pi_{n-3}$ are independent of the exponents. According to one convention, these are the $n-3$ accessory parameters.

The automorphisms of (2.1) include the group of Möbius transformations of the independent variable $x$, i.e., the projective linear group $P G L(2, \mathbb{C})$. If $x=P y \stackrel{\text { def }}{=}$ $(A y+B) /(C y+D)$ with $A D-B C \neq 0$, the equation $T u=0$ for $u=u(x)$ can be pulled back to an equation $\tilde{T} \tilde{u}=0$ for $\tilde{u}=\tilde{u}(y) \stackrel{\text { def }}{=} u(P y)$. This has singular points $y=P^{-1} d_{1}, \ldots, P^{-1} d_{n-1}$ (and also $P^{-1} \infty$, if $\infty$ is a singular point of $T u=0$ ). The exponents are preserved under Möbius transformations, though the accessory 
parameters are not. The exponents of $\widetilde{T} \tilde{u}=0$ at any $y \in \mathbb{P}^{1}(\mathbb{C})$ will be the exponents of $T u=0$ at $x=P y$. This applies to ordinary points as well as singular points. The exponents of any finite ordinary point are 0,1 , and if the point at infinity is ordinary, its exponents will be $0,-1$.

The automorphisms of (2.1) also include a group of transformations, linear in the dependent variable, called F-homotopic or index transformations. For each $k \geqslant 1$, such a transformation is specified by distinct finite points $e_{1}, \ldots, e_{k} \in \mathbb{C}$ and corresponding "index shifts" $s_{1}, \ldots, s_{k} \in \mathbb{C}$. The transformed equation is $\widehat{T} \hat{u}=0$, where $\hat{u}=\hat{u}(x) \stackrel{\text { def }}{=} S(x) u(x)$ and $\widehat{T} \stackrel{\text { def }}{=} S T S^{-1}$, with $S=S(x) \stackrel{\text { def }}{=} \prod_{i=1}^{k}\left(x-e_{i}\right)^{s_{i}}$. By examination, $\widehat{T}$ has the same general form as $T$. In $\widehat{T} \hat{u}=0$, the exponents of each point $x=e_{i}$ are shifted by $s_{i}$ relative to those in $T u=0$, and the exponents of $x=\infty$ are shifted by $-\sum_{i=1}^{k} s_{i}$. In general, the accessory parameters are also altered. For each choice of $e_{1}, \ldots, e_{k}$, the index transformations form an abelian group isomorphic to the additive group $\mathbb{C}^{k}$.

The structure of the space of Fuchsian equations up to index transformations is illuminated by two normalized versions of (2.1), traditionally called reduced forms [17, §20]. The first is the symmetrically reduced form

$$
T_{0} u \stackrel{\text { def }}{=} D_{x}^{2} u+\left[\sum_{i=1}^{n-1} \frac{\frac{1}{4}\left(1-\delta_{i}^{2}\right)}{\left(x-d_{i}\right)^{2}}+\frac{\Pi_{n-3}^{0}(x)}{\prod_{i=1}^{n-1}\left(x-d_{i}\right)}\right] u=0,
$$

which has $\rho_{i}+\hat{\rho}_{i}=1$ for each $i$ and $\rho^{(\infty)}+\hat{\rho}^{(\infty)}=-1$. It is obtained from (2.1) by the index transformation which for each $i$, shifts the pair $\rho_{i}, \hat{\rho}_{i}$ by $\left(1-\rho_{i}-\hat{\rho}_{i}\right) / 2$, and $\rho^{(\infty)}, \hat{\rho}^{(\infty)}$ by $-\sum_{i=1}^{n-1}\left(1-\rho_{i}-\hat{\rho}_{i}\right) / 2$. In (2.2),$\delta_{i}^{2} \stackrel{\text { def }}{=}\left(\rho_{i}-\hat{\rho}_{i}\right)^{2}$. The symmetrically reduced form could be denoted $\mathcal{E}_{\text {sym }}\left(d_{1}, \delta_{1} ; \ldots ; d_{n-1}, \delta_{n-1} ; \infty, \delta^{(\infty)} ; Q\right)$, where $Q$ is the vector of $n-3$ accessory parameters.

The other is the asymmetrically reduced form

$$
T_{1} u \stackrel{\text { def }}{=} D_{x}^{2} u+\left[\sum_{i=1}^{n-1} \frac{1-\rho_{i}}{x-d_{i}}\right] D_{x} u+\left[\frac{\Pi_{n-3}^{1}(x)}{\prod_{i=1}^{n-1}\left(x-d_{i}\right)}\right] u=0,
$$

which has one exponent (by convention $\hat{\rho}_{i}$ ) equal to zero at each finite singular point. It is obtained from (2.1) by an index transformation which shifts each of the $n-1$ pairs $\rho_{i}, \hat{\rho}_{i}$ by either $-\rho_{i}$ or $-\hat{\rho}_{i}$; and also shifts $\rho^{(\infty)}, \hat{\rho}^{(\infty)}$ correspondingly. The reduction is not unique, since in general there are $2^{n-1}$ such index transformations. It can be shown that the leading coefficient of $\Pi_{n-3}^{1}$, i.e., the coefficient of $x^{n-3}$, equals $\rho^{(\infty)} \hat{\rho}^{(\infty)}$. This reduced form could be denoted $\mathcal{E}_{\text {asym }}\left(d_{1}, \rho_{1} ; \ldots ; d_{n-1}, \rho_{n-1} ; \infty, \rho^{(\infty)}, \hat{\rho}^{(\infty)} ; Q\right)$, where only $n$ of the exponent parameters are independent, since they must satisfy Fuchs's relation.

Due to the nonuniqueness of the reduction, an order- $2^{n-1}$ group of index transformations acts on this reduced form. It is generated by the commuting involutions

$$
\left(d_{i}, \rho_{i} ; \infty, \rho^{(\infty)}, \hat{\rho}^{(\infty)}\right) \mapsto\left(d_{i},-\rho_{i} ; \infty, \rho_{i}+\hat{\rho}^{(\infty)}, \rho_{i}+\rho^{(\infty)}\right),
$$

$i=1, \ldots, n-1$, and is isomorphic to $\left(\mathbb{Z}_{2}\right)^{n-1}$. On the level of the unreduced Fuchsian equation (2.1), these involutions negate the exponent differences at the points $x=d_{1}, \ldots, d_{n-1}$. It should be noted that the exponents $\rho^{(\infty)}, \hat{\rho}^{(\infty)}$ are determined by (2.3) only up to interchange; this seeming ambiguity in the action (2.4) will be discussed further below. 
How to quotient out Möbius transformations, in addition to index transformations, will also be discussed. The concept of a signed permutation of the $n$ singular points, where the signs keep track of the negatings of exponent differences, will prove useful. A signed permutation of a set of objects $O_{n}=\{1, \ldots, n\}$ is a bijection $\pi: O_{n} \rightarrow O_{n}$ in which each $i \mapsto \pi(i)$ is accompanied by a "sign". Equivalently, it is a bijection $\pi^{\prime}: O_{n}^{\prime} \rightarrow O_{n}^{\prime}$, where $O_{n}^{\prime}=\{1, \ldots, n\} \cup\{-1, \ldots,-n\}$, that satisfies $\pi^{\prime}(-i)=-\pi^{\prime}(i)$ for all $i$. The group of signed permutations of $O_{n}$ has order $2^{n} n$ ! and is isomorphic to the wreath product $\mathbb{Z}_{2} \succ S_{n}$. Any signed permutation may be written in a sign-annotated version of the disjoint cycle representation used for elements of $S_{n}$. For example, [1 $\left.1_{+} 2_{-} 3_{+}\right]\left[4_{-}\right]$signifies $1 \mapsto 2,2 \mapsto-3,3 \mapsto 1,4 \mapsto-4$. Positively signed 1-cycles may be omitted. A signed permutation is said to be evensigned if its annotated cycle representation has an even number of negative signs. Even-signed permutations form a normal subgroup of index 2, which is isomorphic to a semi-direct product $\left(\mathbb{Z}_{2}\right)^{n-1} \rtimes S_{n}$.

The groups of signed and even-signed permutations of $O_{n}$ are isomorphic to the Coxeter groups $\mathcal{B}_{n}$ (when $n \geqslant 2$ ) and $\mathcal{D}_{n}$ (when $n \geqslant 4$ ), respectively. This makes possible the use of known results on subgroups, cosets, generators, and numbers of elements with given order, etc. The following is a brief explanation of these isomorphisms (cf. 4]). A Coxeter group with generators $\sigma_{1}, \ldots, \sigma_{n}$ is defined by the relations $\sigma_{i}^{2}=1$ for each $i$, and zero or more "braid relations" of the form $\left(\sigma_{i} \sigma_{j}\right)^{c_{i j}}=1$. These may be encoded as a Coxeter graph on the vertex set $\sigma_{1}, \ldots, \sigma_{n}$, with each pair $\sigma_{i}, \sigma_{j}$ joined by $c_{i j}-2$ edges. The nonisomorphic Coxeter groups $\mathcal{A}_{n}(n \geqslant 1)$, $\mathcal{B}_{n}(n \geqslant 2)$, and $\mathcal{D}_{n}(n \geqslant 4)$ are the groups defined, respectively, by (1) $c_{i, i+1}=3$ for each $i \leqslant n-1 ;(2) c_{i, i+1}=3$ for each $i \leqslant n-2$, and $c_{n-1, n}=4$; and (3) $c_{i, i+1}=3$ for each $i \leqslant n-2$, and $c_{n-2, n}=3$. The group $\mathcal{A}_{n}$ turns out to be isomorphic to the symmetric group $S_{n+1}$. It follows from the associated graphs that $\mathcal{D}_{3} \cong \mathcal{A}_{3} \cong S_{4}$; the notation $\mathcal{D}_{3}$ is not standard.

That the group of signed permutations of the $n$-set $O_{n}$ is isomorphic to $\mathcal{B}_{n}$ (when $n \geqslant 2$ ) follows by defining $\sigma_{i}=\left[i_{+}(i+1)_{+}\right]$for $i=1, \ldots, n-1$, and $\sigma_{n}=\left[n_{-}\right]$. Similarly, that the group of even-signed permutations is isomorphic to $\mathcal{D}_{n}$ (when $n \geqslant 4$ ) follows by defining $\sigma_{1}, \ldots, \sigma_{n-1}$ in the same way, and $\sigma_{n}=$ $\left[(n-1)_{-} n_{-}\right]$. Each of the cases $n=1,2,3$ is at least partly degenerate. The groups $\mathcal{B}_{n}, \mathcal{D}_{n}$ are respectively isomorphic, when $n=1$, to $\mathbb{Z}_{2}$ and the trivial group; when $n=2$, to the order-8 dihedral group and the Klein 4-group $\mathbb{Z}_{2} \times \mathbb{Z}_{2}$; and when $n=3$, to an order- 48 group and $\mathcal{A}_{3} \cong S_{4}$. It is easy to see that when $n$ is odd, $\mathcal{B}_{n}$ is isomorphic to $\mathbb{Z}_{2} \times \mathcal{D}_{n}$, where the $\mathbb{Z}_{2}$ factor is generated by [1-] $\cdots\left[n_{-}\right]$. So $\mathcal{B}_{3}$ is isomorphic to $\mathbb{Z}_{2} \times S_{4}$, though this fact will not be used in what follows.

\section{Point configurations}

To study the effects of Möbius transformations on the reduced forms (2.2), (2.3), and their interplay with index transformations, it is helpful to focus first on their effects on the singular points. The action of the Möbius group $P G L(2, \mathbb{C})$ on the complex projective line $\mathbb{P}^{1}(\mathbb{C})$ is sharply 3 -transitive: for any two triples of distinct points $\left(x_{1}, x_{2}, x_{3}\right),\left(x_{1}^{\prime}, x_{2}^{\prime}, x_{3}^{\prime}\right)$, there is a unique $P \in P G L(2, \mathbb{C})$ satisfying $x_{i}^{\prime}=P x_{i}$, $i=1,2,3$. Due to this, it is traditional to assume that the singular points of Fuchsian differential equations with $n \geqslant 3$ include $0,1, \infty$, thereby quotienting out (partially) the action of $P G L(2, \mathbb{C})$. 
If $X=\left\{x_{1}, \ldots, x_{n}\right\}$ is an unordered set of $n$ distinct points, three of which are $0,1, \infty$, there will be exactly $n(n-1)(n-2)$ transformations $P \in P G L(2, \mathbb{C})$ for which $P X$ also has this property. For computational purposes, this is best restated in a form referring to ordered sets of points.

\section{Definition 3.1.}

(1) (a) $X[n] \stackrel{\text { def }}{=}\left[\mathbb{P}^{1}(\mathbb{C})\right]^{n} \backslash \Delta$ is the space of $n$ distinct (labeled) points in $\mathbb{P}^{1}(\mathbb{C})$, where $\Delta$ is the set of configurations with two points coinciding. The group $P G L(2, \mathbb{C})$ acts on $X[n]$ diagonally, i.e., on each point individually, and $S_{n}$ acts on it by permuting points. Since these actions commute, the direct product $P G L(2, \mathbb{C}) \times S_{n}$ also acts on $X[n]$.

(b) When $n \geqslant 3$ and $1 \leqslant i_{1}, i_{2}, i_{3} \leqslant n$ are distinct, $X_{i_{1}, i_{2}, i_{3}}[n] \subset X[n]$ is the space of $n$-tuples satisfying $x_{i_{1}}=0, x_{i_{2}}=1, x_{i_{3}}=\infty$.

(2) $X(n) \stackrel{\text { def }}{=} X[n] / P G L(2, \mathbb{C})$ is the space of $n$ distinct (labeled) points in $\mathbb{P}^{1}(\mathbb{C})$, up to Möbius transformations. When $n \geqslant 3, X(n)$ may be realized as any of the $X_{i_{1}, i_{2}, i_{3}}[n]$, in particular, as $X_{1,2,3}[n]$.

Remark 3.1.1. The relation between $n$-tuples $x_{i_{1}, i_{2}, i_{3}}=\left(x_{1}, \ldots, x_{n}\right) \in X_{i_{1}, i_{2}, i_{3}}[n]$ and $x_{i_{1}^{\prime}, i_{2}^{\prime}, i_{3}^{\prime}}=\left(x_{1}^{\prime}, \ldots, x_{n}^{\prime}\right) \in X_{i_{1}^{\prime}, i_{2}^{\prime}, i_{3}^{\prime}}[n]$, both representing the same point in $X(n)$, is straightforward: $x_{i}^{\prime}=P x_{i}$ for each $i$, where $P \in P G L(2, \mathbb{C})$ is the unique Möbius transformation that takes $x_{i_{1}^{\prime}}, x_{i_{2}^{\prime}}, x_{i_{3}^{\prime}}$ to $0,1, \infty$.

Definition 3.2. For every $\mathfrak{x} \in X_{1,2,3}[n] \subset X[n]$, the associated $n$-point hyperKummer group $\mathfrak{K}_{n}(\mathfrak{x})$ is the subset of the direct product group $P G L(2, \mathbb{C}) \times S_{n}$, acting on $X[n]$, which contains all elements $g$ for which $g \cdot \mathfrak{x} \in X_{1,2,3}[n]$.

Proposition 3.3. For every $\mathfrak{x} \in X_{1,2,3}[n], \mathfrak{K}_{n}(\mathfrak{x})$ is a group isomorphic to $S_{n}$, and the collection of $n$ ! elements $(P, s) \in P G L(2, \mathbb{C}) \times S_{n}$ which $\mathfrak{K}_{n}(\mathfrak{x})$ contains can be generated as follows. Let $\mathfrak{x}=\left(x_{1}, \ldots, x_{n}\right)=\left(0,1, \infty, a_{1}, \ldots, a_{n-3}\right)$. For each choice of distinct $1 \leqslant i_{1}, i_{2}, i_{3} \leqslant n$, there is a unique $P \in P G L(2, \mathbb{C})$ that takes $x_{i_{1}}, x_{i_{2}}, x_{i_{3}}$ to $0,1, \infty$. There are $n(n-1)(n-2)$ such choices. For each such $P$, choose $s \in S_{n}$, a permutation of $1, \ldots, n$, such that $\left(P x_{s(1)}, \ldots, P x_{s(n)}\right) \in X_{1,2,3}[n]$. Since necessarily $s(k)=i_{k}, k=1,2,3$, there are $(n-3)$ ! choices for $s$.

Informally, any $\sigma \in \mathfrak{K}_{n}(\mathfrak{x}) \cong S_{n}$, where $\mathfrak{x}=\left(0,1, \infty, a_{1}, \ldots, a_{n-3}\right)$, acts as the composition of two transformations: (i) the Möbius transformation which, acting diagonally, takes three of these $n$ points to $0,1, \infty$ respectively, and (ii) a permutation of the images of the remaining $n-3$ points under this transformation.

Example 3.4. $n=3$, the hypergeometric case. This case is quite degenerate: $X_{1,2,3}[3]=\{(0,1, \infty)\}$ is a single point, as is $X(3)$. The 3-point hyper-Kummer group, $\mathfrak{K}_{3}((0,1, \infty))<P G L(2, \mathbb{C}) \times S_{3}$, is the traditional Kummer group [18, also called the cross-ratio group. It is based on the six Möbius transformations $x \mapsto f(x)$, with $f(x)$ equal to

$$
x, \quad \frac{x}{x-1}, \quad 1-x, \quad \frac{x-1}{x}, \quad \frac{1}{x}, \quad \frac{1}{1-x} .
$$

These permute $0,1, \infty$, so each must be followed by an appropriate (unique) $s \in S_{3}$, to yield a transformation in $P G L(2, \mathbb{C}) \times S_{3}$ that takes $(0,1, \infty)$ to itself.

Example 3.5. $n=4$, the Heun case. $X_{1,2,3}[4]$ is the space of configurations of the form $(0,1, \infty, a)$, so it is effectively the triply punctured sphere $\mathbb{P}^{1}(\mathbb{C}) \backslash\{0,1, \infty\} \ni a$, 
as is $X(4)$. The 4-point hyper-Kummer group, isomorphic to $S_{4}$, was worked out by Heun and may be called the Heun group. $\mathfrak{K}_{4}((0,1, \infty, a))$ is based on the 24 Möbius transformations $x \mapsto f(x)$, with $f(x)$ equal to

$$
\begin{aligned}
& x, \quad \frac{x}{x-1}, \quad \frac{x}{a}, \quad \frac{x}{x-a}, \quad \frac{(1-a) x}{x-a}, \frac{(a-1) x}{a(x-1)}, \\
& 1-x, \frac{x-1}{x}, \frac{x-1}{x-a}, \frac{x-1}{a-1}, \frac{a(x-1)}{x-a}, \frac{a(x-1)}{(a-1) x}, \\
& \frac{1}{x}, \quad \frac{1}{1-x}, \quad \frac{a}{x}, \quad \frac{a}{a-x}, \quad \frac{a-1}{x-1}, \quad \frac{1-a}{x-a}, \\
& \frac{x-a}{x}, \quad \frac{a-x}{a}, \quad \frac{x-a}{x-1}, \frac{a-x}{a-1}, \frac{x-a}{a(x-1)}, \frac{a-x}{(a-1) x} \text {. }
\end{aligned}
$$

The transformations in the four rows of (3.2) take $0,1, \infty, a$, respectively, to 0 , and in a sense, each permutes $0,1, \infty, a$. What this means is that for any $(0,1, a, \infty) \in$ $X_{1,2,3}[4]$, i.e., any $a \in \mathbb{P}^{1}(\mathbb{C}) \backslash\{0,1, \infty\}$, each takes the unordered set $\{0,1, \infty, a\}$ to a set of the form $\left\{0,1, \infty, a^{\prime}\right\}$. An example is $x \mapsto \frac{x}{x-a}$, which takes $0,1, \infty, a$ to $0, a^{\prime}=\frac{1}{1-a}, 1, \infty$, respectively. This element of $P G L(2, \mathbb{C})$ can be written in cycle notation as $(0)(1 a \infty)$, with the understanding that " 1 is mapped to $a$ " really means " 1 is mapped to $a^{\prime}$ ". It must be followed by an appropriate (unique) permutation $s \in S_{4}$, if it is to take $(0,1, \infty, a) \in X_{1,2,3}[4]$ to $\left(0,1, \infty, a^{\prime}\right) \in X_{1,2,3}[4]$.

The $a$-dependent transformation groups $\mathfrak{K}_{4}((0,1, \infty, a)) \subset P G L(2, \mathbb{C}) \times S_{4}$, each isomorphic to $S_{4}$, can usefully be viewed as arising from the action of a single 4-point hyper-Kummer group isomorphic to $S_{4}$, which may be denoted $\mathfrak{K}_{4}$, on $X_{1,2,3}$ [4]. This is because each formal permutation of $0,1, \infty, a$ gives a map from $a \in \mathbb{P}^{1}(\mathbb{C}) \backslash$ $\{0,1, \infty\}$ to $a^{\prime} \in \mathbb{P}^{1}(\mathbb{C}) \backslash\{0,1, \infty\}$, i.e., from $X_{1,2,3}[4]$ to itself. A similar interpretation is possible for any $n \geqslant 3$, but the $n=4$ case is slightly degenerate, although not so much as the $n=3$ case. By examination, the representation of $S_{4}$ by transformations of $X_{1,2,3}[4]$ is not faithful. The orbit of any $a \in \mathbb{P}^{1}(\mathbb{C}) \backslash\{0,1, \infty\}$ consists of no more than 6 points $a^{\prime} \in \mathbb{P}^{1}(\mathbb{C}) \backslash\{0,1, \infty\}$, rather than 24 . These can be obtained directly from $a$ by $a \mapsto a^{\prime}=f(a)$, with $f$ ranging over the Möbius transformations of (3.1). The reduction of 24 values to 6 arises from the invariance of any a under an order-4 normal subgroup of $\mathfrak{K}_{4} \cong S_{4}$ isomorphic to the Klein 4-group $\mathbb{Z}_{2} \times \mathbb{Z}_{2}$, which comprises the maps $x \mapsto x, \frac{a}{x}, \frac{x-a}{x-1}, \frac{a(x-1)}{x-a}$, or in cycle notation, $(0)(1)(\infty),(0 \infty)(1 a),(0 a)(1 \infty),(01)(a \infty)$. For each, $a^{\prime}=a$.

Generic $a$-orbits contain 6 distinct values $a^{\prime}$, but two nongeneric ones contain fewer: the harmonic (lemniscatic) orbit $\left\{-1, \frac{1}{2}, 2\right\}$ and the equianharmonic orbit $\left\{\frac{1}{2} \pm \mathrm{i} \frac{\sqrt{3}}{2}\right\}$. The names are taken from elliptic function theory [1, chap. 18]. These small orbits contain values of $a$ that are invariant under larger subgroups of $S_{4}$ than the Klein 4-group. Each of $a=-1, \frac{1}{2}, 2$ is fixed by a dihedral group of order 8 , the three groups being conjugate in $S_{4}$. Similarly, each of $a=\frac{1}{2} \pm \mathrm{i} \frac{\sqrt{3}}{2}$ is fixed by the alternating subgroup $A_{4}$.

Example 3.6. $n=5$, a nonclassical case. $X_{1,2,3}[5]$ is the space of 5-point configurations in $\mathbb{P}^{1}(\mathbb{C})$ of the form $(0,1, \infty, a, b)$, so it is effectively the complement of five complex lines in $\mathbb{C}^{2}$, i.e., $\left\{(a, b) \in \mathbb{C}^{2} \mid a \neq 0,1, b \neq 0,1, a \neq b\right\}$, as is $X(5)$. 
However, the action on $X_{1,2,3}[5]$ of the 5-point hyper-Kummer group $\mathfrak{K}_{5}$, isomorphic to $S_{5}$, is difficult to visualize. The $5 !=120$ transformations in this group are too numerous to list, but the following example should clarify their effects on $(a, b)$.

By Proposition 3.3 , the action of each $\sigma \in \mathfrak{K}_{5} \cong S_{5}$ on $(0,1, \infty, a, b)$ is based on a Möbius transformation that maps three of $0,1, \infty, a, b$ to $0,1, \infty$. Suppose $a, b, 0$ are mapped to $0,1, \infty$, respectively. Then this transformation must be $x \mapsto \frac{b(x-a)}{(b-a) x}$. The images of the remaining two points, namely $1, \infty$, will be the transformed coordinates $a^{\prime}, b^{\prime}$; in either order, since the action of $\sigma$ involves a permutation of the remaining points, in addition to the Möbius tranformation. It follows that the action of $\sigma$ on $X_{1,2,3}[5]$ will be either the map $(a, b) \mapsto\left(a^{\prime}, b^{\prime}\right)$ with

$$
a^{\prime}=\frac{b(1-a)}{b-a}, \quad b^{\prime}=\frac{b}{b-a},
$$

or the same with $a^{\prime}, b^{\prime}$ interchanged. The corresponding $\sigma \in \mathfrak{K}_{5} \cong S_{5}$ would be written in cycle notation as $(a 0 \infty b 1)$ and $(a 0 \infty)(1 b)$. The above caveat applies: for instance, in the first, " $\infty$ is mapped to $b$ " really means " $\infty$ is mapped to $b^{\prime}$ ".

The $n=5$ case is not degenerate, unlike $n=3$, 4 . If $(0,1, \infty, a, b) \in X_{1,2,3}[5]$ is "generic", i.e., if $0,1, \infty, a, b \in \mathbb{P}^{1}(\mathbb{C})$ are in general position, then its orbit under $\mathfrak{K}_{5} \cong S_{5}$ will consist of 120 distinct points of the form $\left(0,1, \infty, a^{\prime}, b^{\prime}\right)$. Such an orbit is effectively a set of 120 distinct points $\left(a^{\prime}, b^{\prime}\right)$ in the complement of 5 lines in $\mathbb{C}^{2}$, mentioned above. But there are nongeneric orbits. Any orbit that includes a point $(a, b)$ with $a+b=1$ will contain no more than 60 points, since such a point is left invariant by a $\mathbb{Z}_{2}$-group comprising the maps $(a, b) \mapsto(a, b),(a, b) \mapsto(1-b, 1-a)$, or in cycle notation, $(0)(1)(\infty)(a)(b),(01)(a b)(\infty)$. Each point $\left(a^{\prime}, b^{\prime}\right)$ on such an orbit will be invariant under a $\mathbb{Z}_{2}$-group which is conjugate in $S_{5}$ to this one.

Some nongeneric orbits of this type are even smaller. It can be shown that the orbits of $(a, b)=\left(\frac{1+i}{2}, \frac{1-i}{2}\right),\left(\frac{1+\mathrm{i} \sqrt{3}}{2}, \frac{1-\mathrm{i} \sqrt{3}}{2}\right),\left(\frac{3+\sqrt{5}}{2}, \frac{-1-\sqrt{5}}{2}\right)$ consist of $30,20,12$ points $\left(a^{\prime}, b^{\prime}\right)$, respectively.

\section{Key RESULTS}

The joint effects of Möbius and index transformations on Fuchsian differential equations with $n$ singular points can now be determined. To maintain contact with the classical theory of special functions, three of the singular points will be fixed at $x=0,1, \infty$. That is, the finite singular points $d_{1}, \ldots, d_{n-1}$ will be taken to be $0,1, a_{1}, \ldots, a_{n-3}$. The results of 3 on how a group $\mathfrak{K}_{n} \cong S_{n}$ acts on ordered point configurations of the form $\left(0,1, \infty, a_{1}, \ldots, a_{n-3}\right)$ will then apply.

Definition 4.1. The collection of joint Möbius-index transformations that leave invariant, up to parameter changes, a version of the symmetrically reduced form (2.2) that includes $x=0,1$ among the finite singular points, denoted

$$
\mathcal{E}_{\mathrm{sym}}\left(0, \delta^{(0)} ; 1, \delta^{(1)} ; \infty, \delta^{(\infty)} ; a_{1}, \delta_{1} ; \ldots ; a_{n-3}, \delta_{n-3} ; Q\right),
$$

and a similar restricted version of the asymmetrically reduced form (2.3), denoted

$$
\mathcal{E}_{\text {asym }}\left(0, \rho^{(0)} ; 1, \rho^{(1)} ; \infty, \rho^{(\infty)}, \hat{\rho}^{(\infty)} ; a_{1}, \rho_{1} ; \ldots ; a_{n-3}, \rho_{n-3} ; Q\right),
$$

will be called the automorphism group of symmetrically, resp., asymmetrically, reduced $n$-point Fuchsian differential equations. By convention, permutations of $\left(a_{1}, \delta_{1}\right), \ldots,\left(a_{n-3}, \delta_{n-3}\right)$, resp., $\left(a_{1}, \rho_{1}\right), \ldots,\left(a_{n-3}, \rho_{n-3}\right)$, are included, though interchange of the exponents $\rho^{(\infty)}, \hat{\rho}^{(\infty)}$ at infinity is not. 
Each of these groups acts on the associated parameter space, which is coordinatized by the $n-3$ singular point locations $a_{1}, \ldots, a_{n-3}, n$ (independent) exponent parameters, and $n-3$ accessory parameters.

Theorem 4.2. The automorphism group $G_{\mathrm{sym}}(n)$ of symmetrically reduced n-point Fuchsian differential equations is isomorphic to $S_{n}$.

Proof. If $\mathfrak{x}=\left(0,1, \infty, a_{1}, \ldots, a_{n-3}\right)$ is the set of singular points, any $\sigma \in \mathfrak{K}_{n}(\mathfrak{x}) \cong S_{n}$ consists of a Möbius transformation $P_{\sigma}$ that acts on $\mathfrak{x}$ diagonally, and a subsequent permutation. But under Möbius transformations of the independent variable, characteristic exponents of Fuchsian equations accompany the points at which they are evaluated. This follows from the general theory of differential equations in the complex domain, formalized using the Riemann P-symbol [6, § 15.3]. So under $\sigma$, (4.1) will typically be transformed to another $\mathcal{E}_{\text {sym }}$, with transformed parameters. One case requires special treatment: when $d=P_{\sigma} \infty \neq \infty$. In this case, the sum of the two exponents at the finite singular point $x^{\prime}=d$ and at $x^{\prime}=\infty$ will be -1 and 1, respectively, rather than the other way around, as they should be. So $\sigma$ must be followed by a uniquely determined index transformation, based on $S=S\left(x^{\prime}\right)=x^{\prime}-d$, which will shift the exponents, and yield a proper $\mathcal{E}_{\mathrm{sym}}$.

Theorem 4.3. The automorphism group $G_{\text {asym }}(n)$ of asymmetrically reduced $n$ point Fuchsian differential equations is isomorphic to the Coxeter group $\mathcal{D}_{n}$, the group of even-signed permutations of an $n$-set, i.e., is isomorphic to a semi-direct product of the form $\left(\mathbb{Z}_{2}\right)^{n-1} \rtimes S_{n}$.

Remark 4.3.1. The subgroup $S_{n}$ here is $\mathfrak{K}_{n}$, i.e., the group of Möbius automorphisms, including subsequent permutations of the singular points, and the normal subgroup $\left(\mathbb{Z}_{2}\right)^{n-1}$ is the group of index-transformation automorphisms defined in \$2. In cycle notation they comprise, respectively, all unsigned (or equivalently positively signed) permutations of $0,1, \infty, a_{1}, \ldots, a_{n-3}$, and all even-signed permutations of the form $\left[0_{ \pm}\right]\left[1_{ \pm}\right]\left[\infty_{ \pm}\right]\left[\left(a_{1}\right)_{ \pm}\right] \cdots\left[\left(a_{n-3}\right)_{ \pm}\right]$.

Proof. Each $\sigma \in \mathfrak{K}_{n} \cong S_{n}$ yields an automorphism of (4.2), i.e., of (2.3), which transforms the variable $x$ to $P_{\sigma} x$. If $\sigma(\infty) \neq \infty$, the transformed equation's exponents at $x^{\prime}=d=\sigma(\infty)$ will be $\rho^{(\infty)}, \hat{\rho}^{(\infty)}$, and must be shifted so that one of them is zero, to preserve the form of (4.2). By convention, $\rho^{(\infty)}$ will be chosen. The index transformation based on $S=S\left(x^{\prime}\right)=\left(x^{\prime}-d\right)^{-\rho^{(\infty)}}$ will perform this shift.

As a set, the collection of joint Möbius-index transformations $G_{\text {asym }}(n)$ that leave (2.3) invariant will be the Cartesian product $\left(\mathbb{Z}_{2}\right)^{n-1} \times S_{n}$, since the group of index-transformation automorphisms is isomorphic to $\left(\mathbb{Z}_{2}\right)^{n-1}$. (Any index transformation involves a binary choice at each of the $n-1$ finite singular points.) If $(h, \sigma) \in G_{\text {asym }}(n)$, the index transformation $h$ will be viewed as acting after $\sigma \in S_{n}$.

The structure of $G_{\text {asym }}(n)$ as a group remains to be determined. In resolving this issue, a seemingly minor point will be important. The two exponents $\rho^{(\infty)}, \hat{\rho}^{(\infty)}$ do not appear explicitly in the differential equation (2.3), though they do in the symbolic form (4.2). They are uniquely determined by (2.3) only up to interchange. When determining the group structure of $G_{\text {asym }}(n)$, a convention for ordering this pair, after applying any $\sigma \in S_{n}$ and $h \in\left(\mathbb{Z}_{2}\right)^{n-1}$, will need to be followed.

Any automorphism $(h, \sigma) \in G_{\text {asym }}(n)$ may be written as a signed permutation of the singular points $\left(0,1, \infty, a_{1}, \ldots, a_{n-3}\right)$, i.e., as some $g \in \mathcal{B}_{n}$, by annotating 
the cycle representation of $\sigma$ as follows. If $d$ is any of the $n$ points, then provided $\sigma(d) \neq \infty$, the annotation $\ldots d_{+} \sigma(d) \ldots$, resp., .. $d_{-} \sigma(d) \ldots$, will indicate that $d \mapsto \sigma(d)$ is, resp., is not followed by a negation of the nonzero exponent at $x^{\prime}=\sigma(d)$. When $\sigma(d)=\infty$, the annotation of $\ldots d \infty \ldots$ will have a special meaning. The annotation $\ldots d_{-} \infty \ldots$, as opposed to $\ldots d_{+} \infty \ldots$, will signify that after the index transformation $h$ is performed, the exponent parameters $\rho^{(\infty)}, \hat{\rho}^{(\infty)}$ are interchanged. Such a transposition will be included, if necessary, to ensure even-signedness: an even number of negative signs in the annotated cycle representation of $g$. With this convention, $g \in \mathcal{D}_{n} \triangleleft \mathcal{B}_{n}$.

What remains to be shown is that the bijection between $G_{\text {asym }}(n)$ and $\mathcal{D}_{n}$ is a group isomorphism. The fact that $\mathcal{D}_{n} \cong\left(\mathbb{Z}_{2}\right)^{n-1} \rtimes_{\Gamma} S_{n}$ can be used, where $\Gamma: S_{n} \rightarrow \operatorname{Aut}\left(\left(\mathbb{Z}_{2}\right)^{n-1}\right)$ denotes the action of $S_{n}$ by conjugation. If $\sigma \in S_{n}$ fixes $\infty$, i.e., permutes only the $n-1$ finite singular points, then conjugating $h \in\left(\mathbb{Z}_{2}\right)^{n-1}$ by $\sigma$ will permute the points at which $h$ negates an exponent. So in this case, the conjugation actions on the copies of $\left(\mathbb{Z}_{2}\right)^{n-1}$ in $\mathcal{D}_{n}$ and $G_{\text {asym }}(n)$ are isomorphic.

The harder case is when $\sigma(\infty) \neq \infty$. There are several subcases, but the following example makes clear how each can be handled. Let $\sigma$ be the involution $(0 \infty)$, or in annotated notation $\left[0_{+} \infty_{+}\right]$, and let $h=\left[1_{-}\right]\left[\infty_{-}\right]$. The equation

$$
\left[0_{+} \infty_{+}\right]^{-1} \cdot\left[1_{-}\right]\left[\infty_{-}\right] \cdot\left[0_{+} \infty_{+}\right]=\left[0_{-}\right]\left[1_{-}\right]\left[\infty_{+}\right]
$$

holds in $\mathcal{D}_{n}$, but its validity in $G_{\text {asym }}(n)$ is not obvious. The automorphism labeled by $\left[1_{-}\right]\left[\infty_{-}\right]$negates the exponent parameter at one of the $n-1$ finite singular points, but the conjugated automorphism labeled by $\left[0_{-}\right]\left[1_{-}\right]\left[\infty_{+}\right]$does so at two. The identity of the left side with the right can be verified by sequentially working out the action of the product on the left. Take $G_{\text {asym }}(n)$ to act on (4.2), i.e., on $\mathcal{E}_{\text {asym }}$, 'on the left', so that the order of action of group elements, not previously specified, will be from right to left. Then some straightforward computations reveal that

(1) $\left[0_{+} \infty_{+}\right] \mathcal{E}_{\text {asym }}$, obtained by the Möbius transformation $x \mapsto x^{\prime}=1 / x$ from $\varepsilon_{\text {asym }}$, is of the form

$$
\begin{aligned}
\mathcal{E}_{\text {asym }}\left(0, \hat{\rho}^{(\infty)}-\rho^{(\infty)} ; 1, \rho^{(1)} ; \infty, \rho^{(\infty)}, \rho^{(0)}+\rho^{(\infty)} ;\right. \\
\left.\frac{1}{a_{1}}, \rho_{1} ; \ldots ; \frac{1}{a_{n-3}}, \rho_{n-3} ; Q_{1}\right),
\end{aligned}
$$

with independent variable $x$;

(2) $\left[1_{-}\right]\left[\infty_{-}\right] \cdot\left[0_{+} \infty_{+}\right] \mathcal{E}_{\text {asym }}$, obtained by a further index transformation at $x^{\prime}=1$, is of the form

$$
\begin{array}{r}
\mathcal{E}_{\text {asym }}\left(0, \hat{\rho}^{(\infty)}-\rho^{(\infty)} ; 1,-\rho^{(1)} ; \infty, \rho^{(0)}+\rho^{(1)}+\rho^{(\infty)}, \rho^{(1)}+\rho^{(\infty)} ;\right. \\
\left.\frac{1}{a_{1}}, \rho_{1} ; \ldots ; \frac{1}{a_{n-3}}, \rho_{n-3} ; Q_{2}\right),
\end{array}
$$

with independent variable $x^{\prime}$; and

(3) $\left[0_{+} \infty_{+}\right]^{-1} \cdot\left[1_{-}\right]\left[\infty_{-}\right] \cdot\left[0_{+} \infty_{+}\right] \mathcal{E}_{\text {asym }}$, obtained by a further Möbius transformation $x^{\prime} \mapsto x=1 / x^{\prime}$, is of the form

$$
\begin{aligned}
\mathcal{E}_{\text {asym }}\left(0,-\rho^{(0)} ; 1,-\rho^{(1)} ; \infty, \rho^{(0)}+\rho^{(1)}+\rho^{(\infty)}, \rho^{(0)}+\rho^{(1)}+\hat{\rho}^{(\infty)} ;\right. \\
\left.a_{1}, \rho_{1} ; \ldots ; a_{n-3}, \rho_{n-3} ; Q_{3}\right),
\end{aligned}
$$

with independent variable $x$.

The last of these, with negated exponents at $x=0,1$, clearly has the same exponent parameters as $\left[0_{-}\right]\left[1_{-}\right]\left[\infty_{+}\right] \mathcal{E}_{\text {asym }}$, i.e., as (4.2) acted on by the right side 
of (4.3). The vectors of accessory parameters can also be shown to correspond, by a computation omitted here. This careful sequential approach can be applied in general, to show that conjugation of $\left(\mathbb{Z}_{2}\right)^{n-1}$ by $\sigma \in S_{n}$ always has the same meaning in $G_{\text {asym }}(n)$ as in $\mathcal{D}_{n}$.

Asymmetrically reduced Fuchsian differential equations predominate in applied mathematics, though symmetrically reduced ones are used in conformal mapping. It is usual to express solutions of (4.2) in terms of a canonical local solution: the Frobenius solution at $x=0$ that belongs to the exponent zero, rather than the other exponent $\rho^{(0)}$. It will be denoted

$$
F\left(0, \rho^{(0)} ; 1, \rho^{(1)} ; \infty, \rho^{(\infty)}, \hat{\rho}^{(\infty)} ; a_{1}, \rho_{1} ; \ldots ; a_{n-3}, \rho_{n-3} ; Q ; x\right) .
$$

If $\rho^{(0)}$ is not a positive integer, $F$ is guaranteed to be analytic in a neighborhood of $x=0$, and may be chosen to equal unity at $x=0$.

Proposition 4.4. A family of $2^{n-1} n$ ! formally distinct local solutions of (4.2), indexed by the group $G_{\text {asym }}(n) \cong \mathcal{D}_{n}$, can be generated as follows. Any $g \in G_{\text {asym }}(n)$ will include a Möbius transformation $x \mapsto x^{\prime}=P x$, together with a subsequent permutation of singular points and a subsequent index transformation. Apply g to (4.2), and compute the zero-exponent Frobenius solution of the transformed equation at its singular point $x^{\prime}=0$. This will be of the form $A(x)$ times

$$
F\left(0, \rho^{(0) \prime} ; 1, \rho^{(1) \prime} ; \infty, \rho^{(\infty) \prime}, \hat{\rho}^{(\infty) \prime} ; a_{1}^{\prime}, \rho_{1}^{\prime} ; \ldots ; a_{n-3}^{\prime}, \rho_{n-3}^{\prime} ; Q^{\prime} ; P x\right),
$$

where

$$
A(x)=\text { const } \times x^{\nu^{(0)}}(x-1)^{\nu^{(1)}} \prod_{i=1}^{n-3}\left(x-a_{i}\right)^{\nu_{i}},
$$

and where $\rho^{(0) \prime}, \rho^{(1) \prime}, \rho^{(\infty) \prime}, \hat{\rho}^{(\infty) \prime}, \rho_{1}^{\prime}, \ldots, \rho_{n-3}^{\prime}$ and also $\nu^{(0)}, \nu^{(1)}, \nu_{1}, \ldots, \nu_{n-3}$ are linear functions of $\rho^{(0)}, \rho^{(1)}, \rho^{(\infty)}, \hat{\rho}^{(\infty)}, \rho_{1}, \ldots, \rho_{n-3}$, with coefficients in $\{0, \pm 1\}$.

Proof. Any $g \in G_{\text {asym }}(n)$ is a pair $(h, \sigma) \in\left(\mathbb{Z}_{2}\right)^{n-1} \rtimes S_{n}$, with $\sigma$ acting first. The automorphism $\sigma$ changes the independent variable from $x$ to $x^{\prime}=P_{\sigma} x$, where $P_{\sigma}$ is the associated Möbius transformation. The index transformation $h$ acts by replacing the differential operator $T_{1}$ in the equation by $\widehat{T}_{1}=S T_{1} S^{-1}$, where $S=$ $S\left(x^{\prime}\right)$ is a product over finite transformed singular points of the form $\prod_{i=1}^{n-1}\left(x^{\prime}-d_{i}^{\prime}\right)^{s_{i}^{\prime}}$, with $s_{i}^{\prime}$ the amount by which the exponents at $x^{\prime}=d_{i}^{\prime}$ are shifted. As noted in the proof of Theorem 4.3, if $\sigma(\infty) \neq \infty$, then the automorphism $\sigma$ will contribute an additional factor of the form $\left(x^{\prime}-d_{i}^{\prime}\right)^{s_{i}^{\prime}}$ to the conjugating function $S$. Conjugating $T_{1}$ by the resulting $S$ is equivalent to replacing any solution $u=u\left(x^{\prime}\right)$ of the transformed differential equation, such as $u\left(x^{\prime}\right)=F\left(x^{\prime}\right)=F\left(P_{\sigma} x\right)$, by $S\left(x^{\prime}\right) u\left(x^{\prime}\right)$. Up to a constant factor, $S$ can be written as a product over the untransformed finite singular points, $\prod_{i=1}^{n-1}\left(x-d_{i}\right)^{s_{i}}$, since the transformed singular points are the original singular points, acted on by $P_{\sigma}$ and possibly permuted.

Theorem 4.5. Suppose $g \in G_{\text {asym }, 0}(n) \cong \mathcal{D}_{n-1} \cong\left(\mathbb{Z}_{2}\right)^{n-2} \rtimes S_{n-1}$, the subgroup of $G_{\text {asym }}(n)$ that comprises all automorphisms that fix $x=0$ and perform no index transformation there. That is, in annotated cycle notation $g$ should contain the positive 1-cycle $\left[0_{+}\right]$. Then the local solution of (4.2) obtained from $g$ by the technique of Proposition 4.4, as a function of $x$ near $x=0$, will equal $F$ itself, pointwise. 
Proof. If $g=(h, \sigma)$ with the Möbius transformation $P_{\sigma}$ taking $x=0$ to $x^{\prime}=0$, then $F(P x)$ will be analytic at $x=0$, and moreover, the prefactor $A(x)$ will not include an $x^{\nu^{(0)}}$ factor. So it will belong to the zero exponent at $x=0$, and must be a multiple of $F$ itself.

Corollary 4.6. Of the $2^{n-1} n$ ! formally distinct local solutions of (4.2) generated by the technique of Proposition 4.4, $2^{n-2}(n-1)$ ! solutions (including $F$ ) are equivalent expressions for $F$; and since this collection is bijective with $\mathcal{D}_{n-1}$, the family of transformations of $F$ into any of these alternative forms, which will be called the transformation group of $F$, has a group structure isomorphic to $\mathcal{D}_{n-1}$.

Theorem 4.7. The $2^{n-1} n$ ! local solutions of (4.2) indexed by $G_{\text {asym }}(n) \cong \mathcal{D}_{n}$ split into $2 n$ sets of $2^{n-2}(n-1)$ ! equivalent expressions involving $F$, each set defining one of the two Frobenius solutions at one of the $n$ singular points. These $2 n$ sets are bijective with the cosets of $G_{\text {asym }, 0}(n) \cong \mathcal{D}_{n-1}$ in $G_{\text {asym }}(n) \cong \mathcal{D}_{n}$.

Proof. This is similar to the proof of Theorem 4.5. For any of these $2 n$ sets, the identification of every solution in the set with a single Frobenius solution follows from their identical local behavior at the defining singular point.

A normalization of the $2^{n-1} n$ ! formally distinct local solutions of (4.2) has not yet been chosen. A reasonable one would take each solution defined near a finite singular point $x=d$ with exponent $\rho$ to be asymptotic to $(x-d)^{\rho}$ or $(d-x)^{\rho}$ as $x \rightarrow d$. Similarly, those with exponents $\rho^{(\infty)}, \hat{\rho}^{(\infty)}$ at $x=\infty$ would be taken to be asymptotic to $x^{-\rho^{(\infty)}}, x^{-\hat{\rho}^{(\infty)}}$ as $x \rightarrow \infty$. These conventions assume the exponent difference is not an integer, so that both solutions display power-law behavior.

The following discussion should clarify the action of $G_{\text {asym }}(n) \cong \mathcal{D}_{n}$ on the asymmetrically reduced equation, and in particular, the action of $\mathcal{D}_{n-1}$, the "transformation group of $F$ " on the $2^{n-2}(n-1)$ ! formally distinct expressions for $F$. First, note that the subgroup of $G_{\text {asym }}(n)$ isomorphic to $S_{n}$ contains a subgroup $H \cong S_{n-3}$ that permutes the singular points $a_{1}, \ldots, a_{n-3}$. Clearly, $G_{\text {asym }}(n)>G_{\text {asym }, 0}(n)>H$. The solutions of (4.2) associated to the elements of $H$ are simply $F$ itself, with its $n-3$ argument pairs $\left\{\left(a_{i}, \rho_{i}\right)\right\}$ arbitrarily permuted. So $H$ provides the trivial part of the action of $G_{\text {asym }, 0}(n)$ on the family of equivalent expressions for $F$. When $n \geqslant 5, S_{n-3}$ is not normal in $S_{n}$, so $H$ is not normal in $G_{\text {asym }, 0}(n)$. There is accordingly no natural way of quotienting out these trivial actions.

The following automorphisms act less trivially. Consider the signed permutations $\left[1_{-}\right]\left[\infty_{-}\right]$and $\left[1_{+} \infty_{+}\right]$of the set of singular points $0,1, \infty, a_{1}, \ldots, a_{n-3}$. For a reason explained in the next section, these will be called generalized Euler and Pfaff transformations, respectively. The former performs an index transformation at $x=1$, and the latter "interchanges $x=1$ and $x=\infty$ "; or more accurately, maps $\left(0,1, \infty, a_{1}, \ldots, a_{n-3}\right)$ to $\left(0, \infty, 1, \frac{a_{1}}{a_{1}-1}, \ldots, \frac{a_{n-3}}{a_{n-3}-1}\right)$, since it performs the Möbius transformation $x \mapsto \frac{x}{x-1}$. Each of these implicitly contains the positive 1-cycle $\left[0_{+}\right]$, i.e., each fixes $x=0$ and performs no index transformation there. So each is an element of $G_{\text {asym }, 0}(n)$, and yields an alternative expression for $F$. These are

$$
\begin{array}{r}
F\left(0, \rho^{(0)} ; 1, \rho^{(1)} ; \infty, \rho^{(\infty)}, \hat{\rho}^{(\infty)} ; a_{1}, \rho_{1} ; \ldots ; a_{n-3}, \rho_{n-3} ; Q ; x\right) \\
=(1-x)^{-\rho^{(1)}} F\left(0, \rho^{(0)} ; 1,-\rho^{(1)} ; \infty, \rho^{(1)}+\hat{\rho}^{(\infty)}, \rho^{(1)}+\rho^{(\infty)}\right. \\
\left.a_{1}, \rho_{1} ; \ldots ; a_{n-3}, \rho_{n-3} ; Q^{\prime} ; x\right)
\end{array}
$$




$$
\begin{aligned}
=(1-x)^{-\rho^{(\infty)}} F\left(0, \rho^{(0)} ; 1, \hat{\rho}^{(\infty)}-\rho^{(\infty)} ; \infty, \rho^{(\infty)}, \rho^{(1)}+\rho^{(\infty)} ;\right. \\
\left.\frac{a_{1}}{a_{1}-1}, \rho_{1} ; \ldots ; \frac{a_{n-3}}{a_{n-3}-1}, \rho_{n-3} ; Q^{\prime \prime} ; \frac{x}{x-1}\right),
\end{aligned}
$$

in which the expressions for $Q^{\prime}, Q^{\prime \prime}$, the transformed vectors of accessory parameters, are not given explicitly since they are complicated; especially the latter.

Each of $\left[1_{-}\right]\left[\infty_{-}\right]$and $\left[1_{+} \infty_{+}\right]$is an involution, as are the transformations (4.5) and (4.6) of $F$. They commute, as do (4.5) and (4.6). So they generate a group isomorphic to the Klein 4-group $\mathbb{Z}_{2} \times \mathbb{Z}_{2}$. Besides the identity element, this group contains the signed permutation $\left[1_{-} \infty_{-}\right]$. By examination, the corresponding transformation is the same as (4.6), with $\rho^{(\infty)}$ and $\hat{\rho}^{(\infty)}$ interchanged on both sides, and also with the sixth and seventh arguments of the right-hand $F$ (the exponents at $\infty$ ) interchanged. This transformation is formally different from (4.6) but is equivalent, since the order of the two exponents at $\infty$ is not significant on either side.

This example suggests that for computational purposes, it may be useful to extend the automorphism group to $\mathcal{B}_{n}$, and the transformation group of $F$ to $\mathcal{B}_{n-1}$, by including interchange of the two exponents at infinity. In annotated cycle notation this interchange is the involution $\left[\infty_{-}\right]$, which acts on $\mathcal{D}_{n}$ by conjugation. So $\mathcal{B}_{n} \cong \mathcal{D}_{n} \rtimes \mathbb{Z}_{2}$. To see how computation in $\mathcal{B}_{n-1}$, rather than $\mathcal{D}_{n-1}$, can facilitate the generation of the full set of $2^{n-2}(n-1)$ ! transformations of $F$, consider

$$
\begin{aligned}
{\left[1_{-}\right]\left[\infty_{-}\right] } & =\left(\left[\infty_{-}\right]\right)^{-1}\left[1_{+} \infty_{+}\right]\left(\left[\infty_{-}\right]\right) \cdot\left[1_{+} \infty_{+}\right] \\
& =\left[1_{-} \infty_{-}\right] \cdot\left[1_{+} \infty_{+}\right],
\end{aligned}
$$

as an equality between transformations of $F$. The left side is the generalized Euler transformation (4.5), and the right side is the composition of two transformations: the generalized Pfaff transformation (4.6), which acts first, and the conjugated Pfaff transformation mentioned above. So although the generalized Pfaff transformation is an involution, by computing its twisted composition with itself, one obtains the generalized Euler transformation. The two transformations are subtly related.

\section{The hypergeometric and Heun Cases}

The hypergeometric and Heun equations, (1.1) and (1.2), are the $n=3$ and $n=4$ cases of the asymmetrically reduced equation $(2.3)$ on $\mathbb{P}^{1}(\mathbb{C})$, with the singular points taken to include $0,1, \infty$. These two equations would be written as

$$
\begin{aligned}
& \mathcal{E}_{\text {asym }}(0,1-c ; 1,-a-b+c ; \infty, a, b), \\
& \mathcal{E}_{\text {asym }}(0,1-\gamma ; 1,1-\delta ; \infty, \alpha, \beta ; a, 1-\epsilon ; q),
\end{aligned}
$$

in the notation of $\$ \$ 2$,

For these equations the canonical local solution $F$, i.e., the Frobenius solution at $x=0$ with zero exponent, is defined thus. If $c$ (resp. $\gamma$ ) is a nonpositive integer, then $F$ will typically display logarithmic behavior at $x=0$; that case is not discussed further here. If $c$ (resp. $\gamma$ ) is not a nonpositive integer, then $F$ will be analytic at $x=0$ with a locally convergent series expansion $\sum_{k=0}^{\infty} c_{k} x^{k}$. In the hypergeometric case, $F$ will be the Gauss function ${ }_{2} F_{1}(a, b ; c ; \cdot)$, defined by

$$
(k+1)(k+c) c_{k+1}-(k+a)(k+b) c_{k}=0, \quad k \geqslant 0,
$$

and $c_{0}=1$. The series $\sum_{k=0}^{\infty} c_{k} x^{k}$ will converge on $|x|<1$ to ${ }_{2} F_{1}(a, b ; c ; x)$. In the Heun case, $F$ will be the local Heun function $H l(a, q ; \alpha, \beta, \gamma, \delta ; \cdot)$, defined by [19] 


$$
\begin{aligned}
&(k+1)(k+\gamma) a c_{k+1}-\{k[((k+\gamma+\delta-1) a+(k+\gamma+\epsilon-1)]+q\} \\
&+(k+\alpha-1)(k+\beta-1) c_{k-1}=0, \quad k \geqslant 0,
\end{aligned}
$$

and the initializations $c_{-1}=0, c_{0}=1$. The Heun series $\sum_{k=0}^{\infty} c_{k} x^{k}$ will converge on the disk $|x|<\min (1,|a|)$ to $\operatorname{Hl}(a, q ; \alpha, \beta, \gamma, \delta ; x)$. The exponent parameter $\epsilon$ is not indicated since it is constrained to equal $\alpha+\beta-\gamma-\delta+1$, as noted above.

The technique of Proposition 4.4yields 24 formally distinct local solutions of the hypergeometric equation, indexed by $g \in G_{\text {asym }}(3) \cong \mathcal{D}_{3}$. Each equals ${ }_{2} F_{1}$ with a projectively transformed argument, multiplied by powers of $x$ and $x-1$. It also yields 192 formally distinct local solutions of the Heun equation, indexed by $g \in G_{\text {asym }}(4) \cong \mathcal{D}_{4}$. Each equals $H l$ with a projectively transformed argument, multiplied by powers of $x, x-1$, and $x-a$. The solutions are listed in Tables 1 and 2 respectively, normalized according to the convention introduced in 4.

TABLE 1. Kummer's 24 local solutions of the hypergeometric equation, indexed by $g \in \mathcal{D}_{3}$. They are partitioned into 6 sets of 4 for-

\begin{tabular}{|c|c|}
\hline$g \in \mathcal{D}_{3}$ & Expression corresponding to $g$ \\
\hline \multicolumn{2}{|c|}{ Frobenius solution $\# 1$ at $x=0$ (characteristic exponent zero) } \\
\hline $\begin{array}{l}{\left[0_{+}\right]\left[1_{+}\right]\left[\infty_{+}\right]} \\
{\left[0_{+}\right]\left[1_{-}\right]\left[\infty_{-}\right]}\end{array}$ & $\begin{array}{l}{ }_{2} F_{1}(a, b ; c ; x) \\
(1-x)^{-a-b+c}{ }_{2} F_{1}(c-a, c-b ; c ; x)\end{array}$ \\
\hline$\left[0_{+}\right]\left[1_{+} \infty_{+}\right]$ & $(1-x)^{-a}{ }_{2} F_{1}\left(a, c-b ; c ; \frac{x}{x-1}\right)$ \\
\hline$\left[0_{+}\right]\left[1_{-} \infty_{-}\right]$ & $(1-x)^{-b}{ }_{2} F_{1}\left(c-a, b ; c ; \frac{x}{x-1}\right)$ \\
\hline \multicolumn{2}{|c|}{ Frobenius solution $\# 2$ at $x=0$ (characteristic exponent $1-c)$} \\
\hline$\left[0_{-}\right]\left[1_{+}\right]\left[\infty_{-}\right]$ & $x^{1-c}{ }_{2} F_{1}(b-c+1, a-c+1 ; 2-c ; x)$ \\
\hline$\left[0_{-}\right]\left[1_{-}\right]\left[\infty_{+}\right]$ & $x^{1-c}(1-x)^{-a-b+c}{ }_{2} F_{1}(1-b, 1-a ; 2-c ; x)$ \\
\hline$\left[0_{-}\right]\left[1-\infty_{+}\right]$ & $x^{1-c}(1-x)^{-a+c-1}{ }_{2} F_{1}\left(1-b, a-c+1 ; 2-c ; \frac{x}{x-1}\right)$ \\
\hline$\left[0_{-}\right]\left[1_{+} \infty_{-}\right]$ & $x^{1-c}(1-x)^{-b+c-1}{ }_{2} F_{1}\left(b-c+1,1-a ; 2-c ; \frac{x}{x-1}\right)$ \\
\hline \multicolumn{2}{|c|}{ Frobenius solution $\# 1$ at $x=1$ (characteristic exponent zero) } \\
\hline$\left[1_{+} 0_{+}\right]\left[\infty_{+}\right]$ & ${ }_{2} F_{1}(a, b ; a+b-c+1 ; 1-x)$ \\
\hline$\left[1_{+} 0_{-}\right]\left[\infty_{-}\right]$ & $x^{1-c}{ }_{2} F_{1}(b-c+1, a-c+1 ; a+b-c+1 ; 1-x)$ \\
\hline$\left[1_{+} 0_{+} \infty_{+}\right]$ & $x^{-a}{ }_{2} F_{1}\left(a, a-c+1 ; a+b-c+1 ; \frac{x-1}{x}\right)$ \\
\hline$\left[1_{+} 0_{-} \infty_{-}\right]$ & $x^{-b}{ }_{2} F_{1}\left(b-c+1, b ; a+b-c+1 ; \frac{x-1}{x}\right)$ \\
\hline \multicolumn{2}{|c|}{ Frobenius solution $\# 2$ at $x=1$ (characteristic exponent $-a-b+c$ ) } \\
\hline$\left[1_{-} 0_{+}\right]\left[\infty_{-}\right]$ & $(x-1)^{-a-b+c}{ }_{2} F_{1}(c-a, c-b ;-a-b+c+1 ; 1-x)$ \\
\hline$\left[1_{-} 0_{-}\right]\left[\infty_{+}\right]$ & $x^{1-c}(x-1)^{-a-b+c}{ }_{2} F_{1}(1-b, 1-a ;-a-b+c+1 ; 1-x)$ \\
\hline$\left[10_{-} 0_{-} \infty_{+}\right]$ & $x^{b-c}(x-1)^{-a-b+c}{ }_{2} F_{1}\left(1-b, c-b ;-a-b+c+1 ; \frac{x-1}{x}\right)$ \\
\hline$\left[1_{-} 0_{+} \infty_{-}\right]$ & $x^{a-c}(x-1)^{-a-b+c}{ }_{2} F_{1}\left(c-a, 1-a ;-a-b+c+1 ; \frac{x-1}{x}\right)$ \\
\hline \multicolumn{2}{|c|}{ Frobenius solution \#1 at $x=\infty$ (characteristic exponent a) } \\
\hline$\left[\infty_{+} 0_{+}\right]\left[1_{+}\right]$ & $x^{-a}{ }_{2} F_{1}\left(a, a-c+1 ; a-b+1 ; \frac{1}{x}\right)$ \\
\hline$\left[\infty_{+} 0_{-}\right]\left[1_{-}\right]$ & ${ }^{+c}{ }_{2} F_{1}(1-b, c-b ; a-$ \\
\hline$\left[\infty_{+} 0_{+} 1_{+}\right]$ & $(x-1)^{-a}{ }_{2} F_{1}\left(a, c-b ; a-b+1 ; \frac{1}{1-x}\right)$ \\
\hline$\left[\infty_{+} 0_{-} 1_{-}\right]$ & ${ }_{2}^{-1} F_{1}\left(1-b, a-c+1 ; a-b+1 ; \frac{1}{1-x}\right)$ \\
\hline \multicolumn{2}{|c|}{ Frobenius solution \#2 at $x=\infty$ (characteristic exponent b) } \\
\hline$\left[\infty_{-} 0_{-}\right]\left[1_{+}\right]$ & $x^{-b}{ }_{2} F_{1}\left(b-c+1, b ;-a+b+1 ; \frac{1}{x}\right)$ \\
\hline$\left[\infty 0_{+}\right]\left[1_{-}\right]$ & $x^{a-c}(x-1)^{-a-b+c}{ }_{2} F_{1}\left(c-a, 1-a ;-a+b+1 ; \frac{1}{x}\right)$ \\
\hline$\left[\infty_{-} 0_{+} 1_{-}\right]$ & $(x-1)^{-b}{ }_{2} F_{1}\left(c-a, b ;-a+b+1 ; \frac{1}{1-x}\right)$ \\
\hline$\left[\infty 0_{-} 1_{+}\right]$ & $x^{1-c}(x-1)^{-b+c-1}{ }_{2} F_{1}\left(b-c+1,1-a ;-a+b+1 ; \frac{1}{1-x}\right)$ \\
\hline
\end{tabular}
mally distinct but equivalent expressions. 
TABLE 2. The 192 local solutions of Heun's equation, indexed by $g \in \mathcal{D}_{4}$. They are partitioned into 8 sets of 24 formally distinct but equivalent expressions.

\begin{tabular}{|c|c|}
\hline$g \in \mathcal{D}_{4}$ & Expression corresponding to $g$ \\
\hline & Frobenius solution $\# 1$ at $x=0$ (characteristic exponent zero $)$ \\
\hline$\left[0_{+}\right]\left[1_{+}\right]\left[a_{+}\right]\left[\infty_{+}\right]$ & $H l(a, q ; \alpha, \beta, \gamma, \delta ; x)$ \\
\hline$\left[0_{+}\right]\left[1_{-}\right]\left[a_{+}\right]\left[\infty_{-}\right]$ & $(1-x)^{1-\delta} H l(a, q-(\delta-1) \gamma a ; \beta-\delta+1, \alpha-\delta+1, \gamma, 2-\delta ; x)$ \\
\hline$\left[0_{+}\right]\left[1_{+}\right]\left[a_{-}\right]\left[\infty_{-}\right]$ & $\left(1-\frac{x}{a}\right)^{-\alpha-\beta+\gamma+\delta} H l(a, q-\gamma(\alpha+\beta-\gamma-\delta) ;-\alpha+\gamma+\delta,-\beta+\gamma+\delta, \gamma, \delta ; x)$ \\
\hline$\left[0_{+}\right]\left[1_{-}\right]\left[a_{-}\right]\left[\infty_{+}\right]$ & $(1-x)^{1-\delta}\left(1-\frac{x}{a}\right)^{-\alpha-\beta+\gamma+\delta} H l(a, q-\gamma[(\delta-1) a+\alpha+\beta-\gamma-\delta] ;-\beta+\gamma+1,-\alpha+\gamma+1, \gamma, 2-\delta ; x)$ \\
\hline$\left[0_{+}\right]\left[1_{+} \infty_{+}\right]\left[a_{+}\right]$ & $(1-x)^{-\alpha} H l\left(\frac{a}{a-1}, \frac{-q+\gamma \alpha a}{a-1} ; \alpha, \alpha-\delta+1, \gamma, \alpha-\beta+1 ; \frac{x}{x-1}\right)$ \\
\hline$\left[0_{+}\right]\left[1_{-} \infty_{-}\right]\left[a_{+}\right]$ & $(1-x)^{-\beta} H l\left(\frac{a}{a-1}, \frac{-q+\gamma \beta a}{a-1} ; \beta-\delta+1, \beta, \gamma,-\alpha+\beta+1 ; \frac{x}{x-1}\right)$ \\
\hline$\left[0_{+}\right]\left[1_{-} \infty_{+}\right]\left[a_{-}\right]$ & $(1-x)^{\beta-\gamma-\delta}\left(1-\frac{x}{a}\right)^{-\alpha-\beta+\gamma+\delta} H l\left(\frac{a}{a-1}, \frac{-q-\gamma[(\beta-\gamma-\delta) a-\alpha-\beta+\gamma+\delta]}{a-1} ;-\beta+\gamma+1,-\beta+\gamma+\delta, \gamma, \alpha-\beta+1 ; \frac{x}{x-1}\right)$ \\
\hline$\left[0_{+}\right]\left[1_{+} \infty_{-}\right]\left[a_{-}\right]$ & $(1-x)^{\alpha-\gamma-\delta}\left(1-\frac{x}{a}\right)^{-\alpha-\beta+\gamma+\delta} \operatorname{Hl}\left(\frac{a}{a-1}, \frac{-q-\gamma[(\alpha-\gamma-\delta) a-\alpha-\beta+\gamma+\delta]}{a-1} ;-\alpha+\gamma+\delta,-\alpha+\gamma+1, \gamma,-\alpha+\beta+1 ; \frac{x}{x-1}\right)$ \\
\hline$\left[0_{+}\right]\left[1_{+} a_{+}\right]\left[\infty_{+}\right]$ & $H l\left(\frac{1}{a}, \frac{q}{a} ; \alpha, \beta, \gamma, \alpha+\beta-\gamma-\delta+1 ; \frac{x}{a}\right)$ \\
\hline$\left[0_{+}\right]\left[1_{+} a_{-}\right]\left[\infty_{-}\right]$ & $\left.\chi+\gamma+\delta,-\beta+\gamma+\delta, \gamma,-\alpha-\beta+\gamma+\delta+1 ; \frac{x}{a}\right)$ \\
\hline$\left[0_{+}\right]\left[1_{-} a_{+}\right]\left[\infty_{-}\right]$ & $(1-x)^{1-\delta} H l\left(\frac{1}{a}, \frac{q-(\delta-1) \gamma a}{a} ; \beta-\delta+1, \alpha-\delta+1, \gamma, \alpha+\beta-\gamma-\delta+1 ; \frac{x}{a}\right)$ \\
\hline$\left[0_{+}\right]\left[1_{-} a_{-}\right]\left[\infty_{+}\right]$ & $(1-x)^{1-\delta}\left(1-\frac{x}{a}\right)^{-\alpha-\beta+\gamma+\delta} H l\left(\frac{1}{a}, \frac{q-\gamma[(\delta-1) a+\alpha+\beta-\gamma-\delta]}{a} ;-\beta+\gamma+1,-\alpha+\gamma+1, \gamma,-\alpha-\beta+\gamma+\delta+1 ; \frac{x}{a}\right)$ \\
\hline$\left[0_{+}\right]\left[1_{+} a_{+} \infty_{+}\right]$ & $\left(1-\frac{x}{a}\right)^{-\alpha} H l\left(\frac{1}{1-a}, \frac{q-\gamma \alpha}{a-1} ; \alpha,-\beta+\gamma+\delta, \gamma, \alpha-\beta+1 ; \frac{x}{x-a}\right)$ \\
\hline$\left[0_{+}\right]\left[1_{+} a_{-} \infty_{-}\right]$ & $\left(1-\frac{x}{a}\right)^{-\beta} H l\left(\frac{1}{1-a}, \frac{q-\gamma \beta}{a-1} ;-\alpha+\gamma+\delta, \beta, \gamma,-\alpha+\beta+1 ; \frac{x}{x-a}\right)$ \\
\hline$\left[0_{+}\right]\left[1_{-} a_{-} \infty_{+}\right]$ & $(1-x)^{1-\delta}\left(1-\frac{x}{a}\right)^{-\alpha+\delta-1} H l\left(\frac{1}{1-a}, \frac{q-\gamma[(\delta-1) a+\alpha-\delta+1]}{a-1} ;-\beta+\gamma+1, \alpha-\delta+1, \gamma, \alpha-\beta+1 ; \frac{x}{x-a}\right)$ \\
\hline$\left[0_{+}\right]\left[1_{-} a_{+} \infty_{-}\right]$ & $(1-x)^{1-\delta}\left(1-\frac{x}{a}\right)^{-\beta+\delta-1} H l\left(\frac{1}{1-a}, \frac{q-\gamma[(\delta-1) a+\beta-\delta+1]}{a-1} ; \beta-\delta+1,-\alpha+\gamma+1, \gamma,-\alpha+\beta+1 ; \frac{x}{x-a}\right)$ \\
\hline$\left[0_{+}\right]\left[1_{+}\right]\left[a_{+} \infty_{+}\right]$ & $\left(1-\frac{x}{a}\right)^{-\alpha} H l\left(1-a,-q+\gamma \alpha ; \alpha,-\beta+\gamma+\delta, \gamma, \delta ; \frac{(1-a) x}{x-a}\right)$ \\
\hline$\left[0_{+}\right]\left[1_{-}\right]\left[a_{-} \infty_{+}\right]$ & $(1-x)^{1-\delta}\left(1-\frac{x}{a}\right)^{-\alpha+\delta-1} H l\left(1-a,-q+\gamma[(\delta-1) a+\alpha-\delta+1] ;-\beta+\gamma+1, \alpha-\delta+1, \gamma, 2-\delta ; \frac{(1-a) x}{x-a}\right)$ \\
\hline$\left[0_{+}\right]\left[1_{+}\right]\left[a_{-} \infty_{-}\right]$ & $\left(1-\frac{x}{a}\right)^{-\beta} H l\left(1-a,-q+\gamma \beta ;-\alpha+\gamma+\delta, \beta, \gamma, \delta ; \frac{(1-a) x}{x-a}\right)$ \\
\hline$\left[0_{+}\right]\left[1_{-}\right]\left[a_{+} \infty_{-}\right]$ & $(1-x)^{1-\delta}\left(1-\frac{x}{a}\right)^{-\beta+\delta-1} H l\left(1-a,-q+\gamma[(\delta-1) a+\beta-\delta+1] ; \beta-\delta+1,-\alpha+\gamma+1, \gamma, 2-\delta ; \frac{(1-a) x}{x-a}\right)$ \\
\hline
\end{tabular}




\begin{tabular}{|c|c|}
\hline $\begin{array}{l}{\left[0_{+}\right]\left[1_{-} \infty_{-} a_{+}\right]} \\
{\left[0_{+}\right]\left[1_{+} \infty_{-} a_{-}\right]}\end{array}$ & 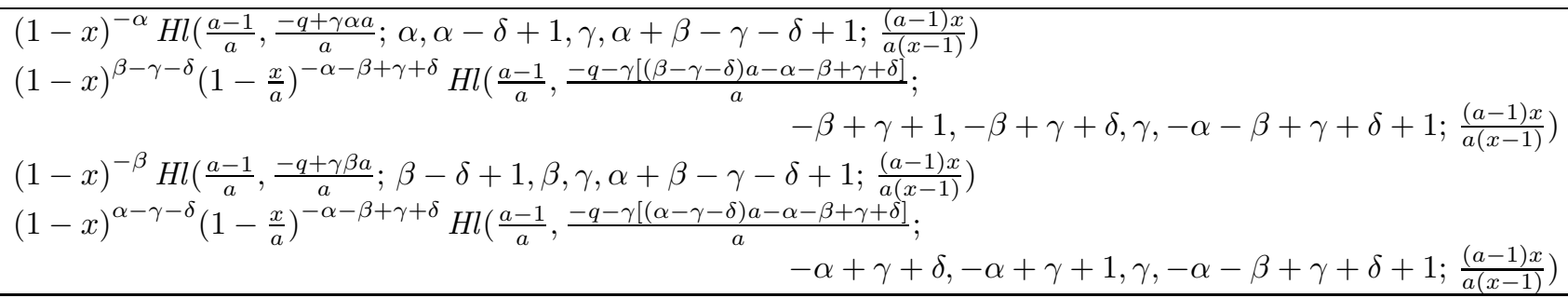 \\
\hline & Fro \\
\hline $\begin{array}{l}{\left[0_{-}\right]\left[1_{+}\right]\left[a_{+}\right]\left[\infty_{-}\right]} \\
{\left[0_{-}\right]\left[1_{-}\right]\left[a_{+}\right]\left[\infty_{+}\right]} \\
{\left[0_{-}\right]\left[1_{+}\right]\left[a_{-}\right]\left[\infty_{+}\right]} \\
{\left[0_{-}\right]\left[1_{-}\right]\left[a_{-}\right]\left[\infty_{-}\right]}\end{array}$ & $\begin{array}{l}x^{1-\gamma} H l(a, q-(\gamma-1)(\delta a+\alpha+\beta- \\
x^{1-\gamma}(1-x)^{1-\delta} H l(a, q-(\gamma+\delta-2) c \\
x^{1-\gamma}\left(1-\frac{x}{a}\right)^{-\alpha-\beta+\gamma+\delta} H l(a, q-\delta(\gamma- \\
x^{1-\gamma}(1-x)^{1-\delta}\left(1-\frac{x}{a}\right)^{-\alpha-\beta+\gamma+\delta} H l(\end{array}$ \\
\hline $\begin{array}{l}{\left[0_{-}\right]\left[1_{+} \infty_{-}\right]\left[a_{+}\right]} \\
{\left[0_{-}\right]\left[1_{+} \infty_{+}\right]\left[a_{-}\right]} \\
{\left[0_{-}\right]\left[1_{-} \infty_{-}\right]\left[a_{-}\right]}\end{array}$ & 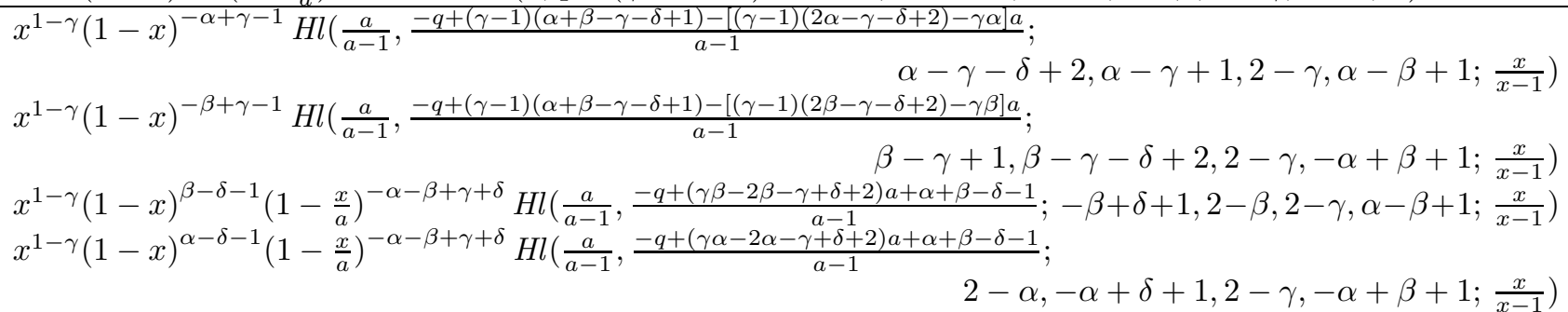 \\
\hline $\begin{array}{l}\left.0_{-}\right]\left[1_{+} a_{+}\right]\left[\infty_{-}\right] \\
\left.0_{-}\right]\left[1_{+} a_{-}\right]\left[\infty_{+}\right] \\
\left.0_{-}\right]\left[1_{-} a_{+}\right]\left[\infty_{+}\right] \\
\left.0_{-}\right]\left[1_{-} a_{-}\right]\left[\infty_{-}\right] \\
\end{array}$ & $\begin{array}{l}x^{1-\gamma} H l\left(\frac{1}{a}, \frac{q-(\gamma-1)(\delta a+\alpha+\beta-\gamma-\delta+1)}{a} ; \beta\right. \\
x^{1-\gamma}\left(1-\frac{x}{a}\right)^{-\alpha-\beta+\gamma+\delta} \operatorname{Hl}\left(\frac{1}{a}, \frac{q-\delta(\gamma-1) a}{}\right. \\
x^{1-\gamma}(1-x)^{1-\delta} H l\left(\frac{1}{a}, \frac{q-\gamma+\delta-2) a-(\gamma-1}{a}\right. \\
x^{1-\gamma}(1-x)^{1-\delta}\left(1-\frac{x}{a}\right)^{-\alpha-\beta+\gamma+\delta} H l\left(\frac{1}{a}\right.\end{array}$ \\
\hline
\end{tabular}




\begin{tabular}{|c|c|}
\hline $\begin{array}{l}{\left[0_{-}\right]\left[1_{+} a_{-} \infty_{+}\right]} \\
{\left[0_{-}\right]\left[1_{+} a_{+} \infty_{-}\right]} \\
{\left[0_{-}\right]\left[1_{-} a_{+} \infty_{+}\right]} \\
{\left[0_{-}\right]\left[1_{-} a_{-} \infty_{-}\right]}\end{array}$ & $\begin{array}{l}x^{1-\gamma}\left(1-\frac{x}{a}\right)^{-\alpha+\gamma-1} H l\left(\frac{1}{1-a}, \frac{q-\delta(\gamma-1) a+(\gamma-1)(\alpha-\beta+\delta+1)-\gamma \alpha}{a-1} ;-\beta+\delta+1, \alpha-\gamma+1,2-\gamma, \alpha-\beta+1 ; \frac{x}{x-a}\right) \\
x^{1-\gamma}\left(1-\frac{x}{a}\right)^{-\beta+\gamma-1} H l\left(\frac{1}{1-a}, \frac{q-\delta(\gamma-1) a-(\gamma-1)(\alpha-\beta-\delta-1)-\gamma \beta}{a-1} ; \beta-\gamma+1,-\alpha+\delta+1,2-\gamma,-\alpha+\beta+1 ; \frac{x}{x-a}\right) \\
x^{1-\gamma}(1-x)^{1-\delta}\left(1-\frac{x}{a}\right)^{-\alpha+\gamma+\delta-2} H l\left(\frac{1}{1-a}, \frac{q-(\gamma+\delta-2) a-\alpha-\gamma \beta+\beta+2 \gamma+\delta-3}{a-1} ; \alpha-\gamma-\delta+2,2-\beta, 2-\gamma, \alpha-\beta+1 ; \frac{x}{x-a}\right) \\
x^{1-\gamma}(1-x)^{1-\delta}\left(1-\frac{x}{a}\right)^{-\beta+\gamma+\delta-2} H l\left(\frac{1}{1-a}, \frac{q-(\gamma+\delta-2) a-\gamma \alpha+\alpha-\beta+2 \gamma+\delta-3}{a-1} ; 2-\alpha, \beta-\gamma-\delta+2,2-\gamma,-\alpha+\beta+1 ; \frac{x}{x-a}\right)\end{array}$ \\
\hline$\left[0_{-}\right]\left[1_{+}\right]\left[a_{-} \infty_{+}\right]$ & $\begin{array}{c}x^{1-\gamma}\left(1-\frac{x}{a}\right)^{-\alpha+\gamma-1} \operatorname{Hl}(1-a,-q+\delta(\gamma-1) a-(\gamma-1)(\alpha-\beta+\delta+1)+\gamma \alpha ; \\
\left.-\beta+\delta+1, \alpha-\gamma+1,2-\gamma, \delta ; \frac{(1-a) x}{x-a}\right) \\
x^{1-\gamma}(1-x)^{1-\delta}\left(1-\frac{x}{a}\right)^{-\alpha+\gamma+\delta-2} H l(1-a,-q+(\gamma+\delta-2) a+\alpha+\gamma \beta-\beta-2 \gamma-\delta+3 ; \\
\left.\alpha-\gamma-\delta+2,2-\beta, 2-\gamma, 2-\delta ; \frac{(1-a) x}{x-a}\right) \\
x^{1-\gamma}\left(1-\frac{x}{a}\right)^{-\beta+\gamma-1} \operatorname{Hl}(1-a,-q+\delta(\gamma-1) a+(\gamma-1)(\alpha-\beta-\delta-1)+\gamma \beta ; \\
\left.\beta-\gamma+1,-\alpha+\delta+1,2-\gamma, \delta ; \frac{(1-a) x}{x-a}\right) \\
x^{1-\gamma}(1-x)^{1-\delta}\left(1-\frac{x}{a}\right)^{-\beta+\gamma+\delta-2} \operatorname{Hl}(1-a,-q+(\gamma+\delta-2) a+\gamma \alpha-\alpha+\beta-2 \gamma-\delta+3 ; \\
\left.2-\alpha, \beta-\gamma-\delta+2,2-\gamma, 2-\delta ; \frac{(1-a) x}{x-a}\right)\end{array}$ \\
\hline$\left[0_{-}\right]\left[1_{-} \infty\right.$ & 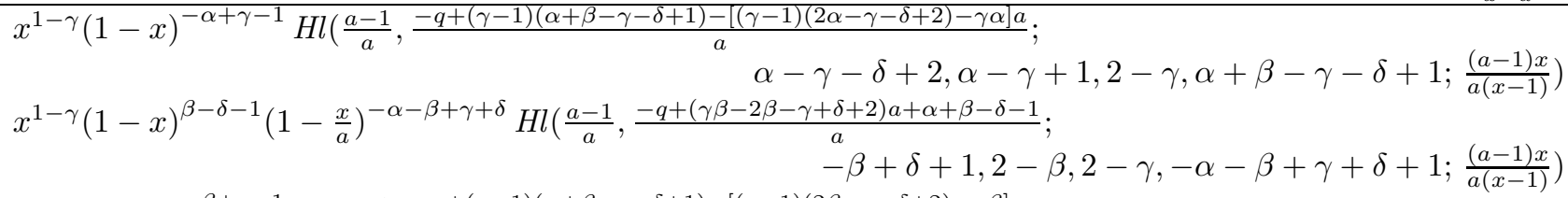 \\
\hline$\left[0_{-}\right]\left[1_{+} \infty\right.$ & $\begin{array}{l}x^{1-\gamma}(1-x)^{-\beta+\gamma-1} H l\left(\frac{a-1}{a}, \frac{-q+(\gamma-1)(\alpha+\beta-\gamma-\delta+1)-[(\gamma-1)(2 \beta-\gamma-\delta+2)-\gamma \beta] a}{a} ;\right. \\
x^{1-\gamma}(1-x)^{\alpha-\delta-1}\left(1-\frac{x}{a}\right)^{-\alpha-\beta+\gamma+\delta} H l\left(\frac{a-1}{a}, \frac{-q+(\gamma \alpha-2 \alpha-\gamma+\delta+2) a+\alpha+\beta-\delta-1}{a} ;\right. \\
\left.2-\alpha,-\alpha+\delta+1,2-\gamma,-\alpha-\beta+\gamma+\delta+1 ; \frac{(a-1) x}{a(x-1)}\right)\end{array}$ \\
\hline
\end{tabular}




\begin{tabular}{|c|c|}
\hline \multicolumn{2}{|r|}{ Frobenius solution $\# 1$ at $x=1$ (characteristic exponent zero) } \\
\hline $\begin{array}{l}{\left[1_{+} 0_{+}\right]\left[a_{+}\right]\left[\infty_{+}\right]} \\
{\left[1_{+} 0_{-}\right]\left[a_{+}\right]\left[\infty_{-}\right]} \\
{\left[1_{+} 0_{+}\right]\left[a_{-}\right]\left[\infty_{-}\right]} \\
{\left[1_{+} 0_{-}\right]\left[a_{-}\right]\left[\infty_{+}\right]}\end{array}$ & $\begin{array}{l}\mathrm{Hl}(1-a,-q+\beta \alpha ; \alpha, \beta, \delta, \gamma ; 1-x) \\
x^{1-\gamma} H l(1-a,-q+\delta(\gamma-1) a+\beta \alpha-\delta \gamma+\delta ; \beta-\gamma+1, \alpha-\gamma+1, \delta, 2-\gamma ; 1-x) \\
\left(\frac{x-a}{1-a}\right)^{-\alpha-\beta+\gamma+\delta} H l(1-a,-q+\beta \alpha-\delta(\alpha+\beta-\gamma-\delta) ;-\alpha+\gamma+\delta,-\beta+\gamma+\delta, \delta, \gamma ; 1-x) \\
x^{1-\gamma}\left(\frac{x-a}{1-a}\right)^{-\alpha-\beta+\gamma+\delta} H l(1-a,-q+\delta(\gamma-1) a-\delta(\alpha+\beta-\delta-1)+\beta \alpha ; \\
\quad-\beta+\delta+1,-\alpha+\delta+1, \delta, 2-\gamma ; 1-x)\end{array}$ \\
\hline $\begin{array}{l}{\left[1_{+} 0_{+} \infty_{+}\right]\left[a_{+}\right]} \\
{\left[1_{+} 0_{-} \infty_{-}\right]\left[a_{+}\right]} \\
{\left[1_{+} 0_{-} \infty_{+}\right]\left[a_{-}\right]} \\
{\left[1_{+} 0_{+} \infty_{-}\right]\left[a_{-}\right]} \\
\end{array}$ & $\begin{array}{l}x^{-\alpha} \operatorname{Hl}\left(\frac{a-1}{a}, \frac{-q+\alpha(\delta a+\beta-\delta)}{a} ; \alpha, \alpha-\gamma+1, \delta, \alpha-\beta+1 ; \frac{x-1}{x}\right) \\
x^{-\beta} \operatorname{Hl}\left(\frac{a-1}{a}, \frac{-q+\beta(\delta a+\alpha-\delta)}{a} ; \beta-\gamma+1, \beta, \delta,-\alpha+\beta+1 ; \frac{x-1}{x}\right) \\
x^{\beta-\gamma-\delta}\left(\frac{x-a}{1-a}\right)^{-\alpha-\beta+\gamma+\delta} \operatorname{Hl}\left(\frac{a-1}{a}, \frac{-q+\beta-\delta) \alpha-\delta(\beta-\gamma-\delta) a}{a} ;-\beta+\delta+1,-\beta+\gamma+\delta, \delta, \alpha-\beta+1 ; \frac{x-1}{x}\right) \\
x^{\alpha-\gamma-\delta}\left(\frac{x-a}{1-a}\right)^{-\alpha-\beta+\gamma+\delta} \operatorname{Hl}\left(\frac{a-1}{a}, \frac{-q+\beta(\alpha-\delta)-\delta(\alpha-\gamma-\delta) a}{a} ;-\alpha+\gamma+\delta,-\alpha+\delta+1, \delta,-\alpha+\beta+1 ; \frac{x-1}{x}\right) \\
\end{array}$ \\
\hline $\begin{array}{l}{\left[1_{+} 0_{+} a_{+} \infty_{+}\right]} \\
{\left[1_{+} 0_{+} a_{-} \infty_{-}\right]} \\
{\left[1_{+} 0_{-} a_{-} \infty_{+}\right]} \\
{\left[1_{+} 0_{-} a_{+} \infty_{-}\right]}\end{array}$ & $\begin{array}{l}\left(\frac{x-a}{1-a}\right)^{-\alpha} \operatorname{Hl}\left(\frac{1}{a}, \frac{q-(\beta-\delta) \alpha}{a} ; \alpha,-\beta+\gamma+\delta, \delta, \alpha-\beta+1 ; \frac{x-1}{x-a}\right) \\
\left(\frac{x-a}{1-a}\right)^{-\beta} \operatorname{Hl}\left(\frac{1}{a}, \frac{q-\beta(\alpha-\delta)}{a} ;-\alpha+\gamma+\delta, \beta, \delta,-\alpha+\beta+1 ; \frac{x-1}{x-a}\right) \\
x^{1-\gamma}\left(\frac{x-a}{1-a}\right)^{-\alpha+\gamma-1} \operatorname{Hl}\left(\frac{1}{a}, \frac{q-\delta(\gamma-1) a-(\beta-\delta) \alpha}{a} ;-\beta+\delta+1, \alpha-\gamma+1, \delta, \alpha-\beta+1 ; \frac{x-1}{x-a}\right) \\
x^{1-\gamma}\left(\frac{x-a}{1-a}\right)^{-\beta+\gamma-1} \operatorname{Hl}\left(\frac{1}{a}, \frac{q-\delta(\gamma-1) a-\beta(\alpha-\delta)}{a} ; \beta-\gamma+1,-\alpha+\delta+1, \delta,-\alpha+\beta+1 ; \frac{x-1}{x-a}\right) \\
\end{array}$ \\
\hline $\begin{array}{l}{\left[1_{+} 0_{+} a_{+}\right]\left[\infty_{+}\right]} \\
{\left[1_{+} 0_{+} a_{-}\right]\left[\infty_{-}\right]} \\
{\left[1_{+} 0_{-} a_{+}\right]\left[\infty_{-}\right]} \\
{\left[1_{+} 0_{-} a_{-}\right]\left[\infty_{+}\right]}\end{array}$ & $\begin{array}{l}H l\left(\frac{1}{1-a}, \frac{q-\beta \alpha}{a-1} ; \alpha, \beta, \delta, \alpha+\beta-\gamma-\delta+1 ; \frac{x-1}{a-1}\right) \\
\left(\frac{x-a}{1-a}\right)^{-\alpha-\beta+\gamma+\delta} H l\left(\frac{1}{1-a}, \frac{q+\delta(\alpha+\beta-\gamma-\delta)-\beta \alpha}{a-1} ;-\alpha+\gamma+\delta,-\beta+\gamma+\delta, \delta,-\alpha-\beta+\gamma+\delta+1 ; \frac{x-1}{a-1}\right) \\
x^{1-\gamma} H l\left(\frac{1}{1-a}, \frac{q-\delta(\gamma-1) a-\beta \alpha+\delta \gamma-\delta}{a-1} ; \beta-\gamma+1, \alpha-\gamma+1, \delta, \alpha+\beta-\gamma-\delta+1 ; \frac{x-1}{a-1}\right) \\
x^{1-\gamma}\left(\frac{x-a}{1-a}\right)^{-\alpha-\beta+\gamma+\delta} H l\left(\frac{1}{1-a}, \frac{q-\delta(\gamma-1) a+\delta(\alpha+\beta-\delta-1)-\beta \alpha}{a-1} ;-\beta+\delta+1,-\alpha+\delta+1, \delta,-\alpha-\beta+\gamma+\delta+1 ; \frac{x-1}{a-1}\right)\end{array}$ \\
\hline $\begin{array}{l}{\left[1_{+} 0_{+}\right]\left[a_{+} \infty_{+}\right]} \\
{\left[1_{+} 0_{-}\right]\left[a_{-} \infty_{+}\right]} \\
{\left[1_{+} 0_{+}\right]\left[a_{-} \infty_{-}\right]} \\
{\left[1_{+} 0_{-}\right]\left[a_{+} \infty_{-}\right]}\end{array}$ & $\begin{array}{l}\left(\frac{x-a}{1-a}\right)^{-\alpha} H l\left(a, q-(\beta-\delta) \alpha ; \alpha,-\beta+\gamma+\delta, \delta, \gamma ; \frac{a(x-1)}{x-a}\right) \\
x^{1-\gamma}\left(\frac{x-a}{1-a}\right)^{-\alpha+\gamma-1} H l\left(a, q-\delta(\gamma-1) a-(\beta-\delta) \alpha ;-\beta+\delta+1, \alpha-\gamma+1, \delta, 2-\gamma ; \frac{a(x-1)}{x-a}\right) \\
\left(\frac{x-a}{1-a}\right)^{-\beta} H l\left(a, q-\beta(\alpha-\delta) ;-\alpha+\gamma+\delta, \beta, \delta, \gamma ; \frac{a(x-1)}{x-a}\right) \\
x^{1-\gamma}\left(\frac{x-a}{1-a}\right)^{-\beta+\gamma-1} H l\left(a, q-\delta(\gamma-1) a-\beta(\alpha-\delta) ; \beta-\gamma+1,-\alpha+\delta+1, \delta, 2-\gamma ; \frac{a(x-1)}{x-a}\right)\end{array}$ \\
\hline
\end{tabular}




\begin{tabular}{|c|c|}
\hline $\begin{array}{l}{\left[1_{+} 0_{+} \infty_{+} a_{+}\right]} \\
{\left[1_{+} 0_{-} \infty_{+} a_{-}\right]} \\
{\left[1_{+} 0_{-} \infty_{-} a_{+}\right]} \\
{\left[1_{+} 0_{+} \infty_{-} a_{-}\right]}\end{array}$ & $\begin{array}{l}x^{-\alpha} H l\left(\frac{a}{a-1}, \frac{-q+\alpha(\delta a+\beta-\delta)}{a-1} ; \alpha, \alpha-\gamma+1, \delta, \alpha+\beta-\gamma-\delta+1 ; \frac{a(x-1)}{(a-1) x}\right) \\
x^{\beta-\gamma-\delta}\left(\frac{x-a}{1-a}\right)^{-\alpha-\beta+\gamma+\delta} \operatorname{Hl}\left(\frac{a}{a-1}, \frac{-q+(\beta-\delta) \alpha-\delta(\beta-\gamma-\delta) a}{a-1} ;-\beta+\delta+1,-\beta+\gamma+\delta, \delta,-\alpha-\beta+\gamma+\delta+1 ; \frac{a(x-1)}{(a-1) x}\right) \\
x^{-\beta} H l\left(\frac{a}{a-1}, \frac{-q+\beta(\delta a+\alpha-\delta)}{a-1} ; \beta-\gamma+1, \beta, \delta, \alpha+\beta-\gamma-\delta+1 ; \frac{a(x-1)}{(a-1) x}\right) \\
x^{\alpha-\gamma-\delta}\left(\frac{x-a}{1-a}\right)^{-\alpha-\beta+\gamma+\delta} \operatorname{Hl}\left(\frac{a}{a-1}, \frac{-q+\beta(\alpha-\delta)-\delta(\alpha-\gamma-\delta) a}{a-1} ;-\alpha+\gamma+\delta,-\alpha+\delta+1, \delta,-\alpha-\beta+\gamma+\delta+1 ; \frac{a(x-1)}{(a-1) x}\right)\end{array}$ \\
\hline & Frobenius solution \#2 at $x=1$ (characteristic exponent $1-\delta$ ) \\
\hline $\begin{array}{l}{\left[1_{-} 0_{+}\right]\left[a_{+}\right]\left[\infty_{-}\right]} \\
{\left[1_{-} 0_{-}\right]\left[a_{+}\right]\left[\infty_{+}\right]} \\
{\left[1_{-} 0_{+}\right]\left[a_{-}\right]\left[\infty_{+}\right]}\end{array}$ & $\begin{array}{c}\begin{array}{r}(x-1)^{1-\delta} H l(1-a,-q+(\delta-1) \gamma a+(\beta-\delta+1)(\alpha-\delta+1) ; \beta-\delta+1, \alpha-\delta+1,2-\delta, \gamma ; 1-x) \\
x^{1-\gamma}(x-1)^{1-\delta} H l(1-a,-q+(\gamma+\delta-2) a-\delta(\alpha+\beta-\gamma-\delta+1)+\beta \alpha+\alpha+\beta-2 \gamma-2 \delta+3 ; \\
\alpha-\gamma-\delta+2, \beta-\gamma-\delta+2,2-\delta, 2-\gamma ; 1-x) \\
(x-1)^{1-\delta}\left(\frac{x-a}{1-a}\right)^{-\alpha-\beta+\gamma+\delta} H l(1-a,-q+(\delta-1) \gamma a+\beta \alpha-\alpha-\beta-\delta \gamma+2 \gamma+1 ; \\
-\beta+\gamma+1,-\alpha+\gamma+1,2-\delta, \gamma ; 1-x) \\
x^{1-\gamma}(x-1)^{1-\delta}\left(\frac{x-a}{1-a}\right)^{-\alpha-\beta+\gamma+\delta} H l(1-a,-q+(\gamma+\delta-2) a+\beta \alpha-\alpha-\beta-\delta+3 ; \\
2-\alpha, 2-\beta, 2-\delta, 2-\gamma ; 1-x)\end{array}\end{array}$ \\
\hline $\begin{array}{l}{\left[1_{-} 0_{-} \infty_{+}\right]\left[a_{+}\right]} \\
{\left[1_{-} 0_{+} \infty_{-}\right]\left[a_{+}\right]} \\
{\left[1_{-} 0_{+} \infty_{+}\right]\left[a_{-}\right]}\end{array}$ & $\begin{array}{l}x^{-\alpha+\delta-1}(x-1)^{1-\delta} \operatorname{Hl}\left(\frac{a-1}{a}, \frac{-q+(\beta-1)(\alpha-\delta+1)-[(\gamma+\delta-2)(\alpha-\delta+1)-\gamma \alpha] a}{a} ; \alpha-\gamma-\delta+2, \alpha-\delta+1,2-\delta, \alpha-\beta+1 ; \frac{x-1}{x}\right) \\
x^{-\beta+\delta-1}(x-1)^{1-\delta} \operatorname{Hl}\left(\frac{a-1}{a}, \frac{-q+(\beta-\delta+1)(\alpha-1)-[(\gamma+\delta-2)(\beta-\delta+1)-\gamma \beta] a}{a} ; \beta-\delta+1, \beta-\gamma-\delta+2,2-\delta,-\alpha+\beta+1 ; \frac{x-1}{x}\right) \\
x^{\beta-\gamma-1}(x-1)^{1-\delta}\left(\frac{x-a}{1-a}\right)^{-\alpha-\beta+\gamma+\delta} \operatorname{Hl}\left(\frac{a-1}{a}, \frac{-q+(\delta \beta-2 \beta+\gamma-\delta+2) a+(\beta-1)(\alpha-\delta+1)}{a} ;\right. \\
\begin{aligned}-\beta+\gamma+1,2-\beta, 2-\delta, \alpha-\beta+1 ; & \left.\frac{x-1}{x}\right) \\
x^{\alpha-\gamma-1}(x-1)^{1-\delta}\left(\frac{x-a}{1-a}\right)^{-\alpha-\beta+\gamma+\delta} H l\left(\frac{a-1}{a}, \frac{-q+(\delta \alpha-2 \alpha+\gamma-\delta+2) a+(\beta-\delta+1)(\alpha-1)}{a} ;\right. & \left.2-\alpha,-\alpha+\gamma+1,2-\delta,-\alpha+\beta+1 ; \frac{x-1}{x}\right)\end{aligned}\end{array}$ \\
\hline $\begin{array}{l}{\left[1_{-} 0_{+} a_{-} \infty_{+}\right]} \\
{\left[1_{-} 0_{+} a_{+} \infty_{-}\right]} \\
{\left[10_{-} 0_{-} a_{+} \infty_{+}\right]} \\
{\left[1_{-} 0_{-} a_{-} \infty_{-}\right]}\end{array}$ & $\begin{array}{l}(x-1)^{1-\delta}\left(\frac{x-a}{1-a}\right)^{-\alpha+\delta-1} \operatorname{Hl}\left(\frac{1}{a}, \frac{q-(\delta-1) \gamma a-(\beta-1)(\alpha-\delta+1)}{a} ;-\beta+\gamma+1, \alpha-\delta+1,2-\delta, \alpha-\beta+1 ; \frac{x-1}{x-a}\right) \\
(x-1)^{1-\delta}\left(\frac{x-a}{1-a}\right)^{-\beta+\delta-1} \operatorname{Hl}\left(\frac{1}{a}, \frac{q-(\delta-1) \gamma a-(\beta-\delta+1)(\alpha-1)}{a} ; \beta-\delta+1,-\alpha+\gamma+1,2-\delta,-\alpha+\beta+1 ; \frac{x-1}{x-a}\right) \\
x^{1-\gamma}(x-1)^{1-\delta}\left(\frac{x-a}{1-a}\right)^{-\alpha+\gamma+\delta-2} \operatorname{Hl}\left(\frac{1}{a}, \frac{q-(\gamma+\delta-2) a-(\beta-1)(\alpha-\delta+1)}{a} ; \alpha-\gamma-\delta+2,2-\beta, 2-\delta, \alpha-\beta+1 ; \frac{x-1}{x-a}\right) \\
x^{1-\gamma}(x-1)^{1-\delta}\left(\frac{x-a}{1-a}\right)^{-\beta+\gamma+\delta-2} \operatorname{Hl}\left(\frac{1}{a}, \frac{q-(\gamma+\delta-2) a-(\beta-\delta+1)(\alpha-1)}{a} ; 2-\alpha, \beta-\gamma-\delta+2,2-\delta,-\alpha+\beta+1 ; \frac{x-1}{x-a}\right)\end{array}$ \\
\hline
\end{tabular}




\begin{tabular}{|c|c|}
\hline $\begin{array}{l}{\left[1_{-} 0_{+} a_{+}\right]\left[\infty_{-}\right]} \\
{\left[1_{-} 0_{+} a_{-}\right]\left[\infty_{+}\right]}\end{array}$ & $\begin{array}{l}(x-1)^{1-\delta} H l\left(\frac{1}{1-a}, \frac{q-(\delta-1) \gamma a-(\beta-\delta+1)(\alpha-\delta+1)}{a-1} ; \beta-\delta+1, \alpha-\delta+1,2-\delta, \alpha+\beta-\gamma-\delta+1 ; \frac{x-1}{a-1}\right) \\
(x-1)^{1-\delta}\left(\frac{x-a}{1-a}\right)^{-\alpha-\beta+\gamma+\delta} \operatorname{Hl}\left(\frac{1}{1-a}, \frac{q-(\delta-1) \gamma a-\beta \alpha+\alpha+\beta+\delta-2 \gamma-1}{a-1} ;\right. \\
x^{1-\gamma}(x-1)^{1-\delta} H l\left(\frac{1}{1-a}, \frac{q-(\gamma+\delta-2) a+\delta(\alpha+\beta-\gamma-\delta+1)-\beta \alpha-\alpha-\beta+2 \gamma+2 \delta-3}{a-1} ;\right. \\
\left.\quad \alpha-\gamma-\delta+2, \beta-\gamma-\delta+2,2-\delta, \alpha+\beta-\gamma-\delta+1 ; \frac{x-1}{a-1}\right) \\
x^{1-\gamma}(x-1)^{1-\delta}\left(\frac{x-a}{1-a}\right)^{-\alpha-\beta+\gamma+\delta} H l\left(\frac{1}{1-a}, \frac{q-(\gamma+\delta-2) a-\beta \alpha+\alpha+\beta+\delta-3}{a-1} ; 2-\alpha, 2-\beta, 2-\delta,-\alpha-\beta+\gamma+\delta+1 ; \frac{x-1}{a-1}\right)\end{array}$ \\
\hline $\begin{array}{l}{\left[1_{-} 0_{+}\right]\left[a_{-} \infty_{+}\right]} \\
{\left[1_{-} 0_{-}\right]\left[a_{+} \infty_{+}\right]}\end{array}$ & $\begin{array}{c}(x-1)^{1-\delta}\left(\frac{x-a}{1-a}\right)^{-\alpha+\delta-1} \operatorname{Hl}\left(a, q-(\delta-1) \gamma a-(\beta-1)(\alpha-\delta+1) ;-\beta+\gamma+1, \alpha-\delta+1,2-\delta, \gamma ; \frac{a(x-1)}{x-a}\right) \\
x^{1-\gamma}(x-1)^{1-\delta}\left(\frac{x-a}{1-a}\right)^{-\alpha+\gamma+\delta-2} \operatorname{Hl}(a, q-(\gamma+\delta-2) a-(\beta-1)(\alpha-\delta+1) ; \\
\left.\quad \alpha-\gamma-\delta+2,2-\beta, 2-\delta, 2-\gamma ; \frac{a(x-1)}{x-a}\right) \\
(x-1)^{1-\delta}\left(\frac{x-a}{1-a}\right)^{-\beta+\delta-1} \operatorname{Hl}\left(a, q-(\delta-1) \gamma a-(\beta-\delta+1)(\alpha-1) ; \beta-\delta+1,-\alpha+\gamma+1,2-\delta, \gamma ; \frac{a(x-a)}{x-a}\right) \\
x^{1-\gamma}(x-1)^{1-\delta}\left(\frac{x-a}{1-a}\right)^{-\beta+\gamma+\delta-2} \operatorname{Hl}(a, q-(\gamma+\delta-2) a-(\beta-\delta+1)(\alpha-1) ; \\
\left.\quad 2-\alpha, \beta-\gamma-\delta+2,2-\delta, 2-\gamma ; \frac{a(x-1)}{x-a}\right)\end{array}$ \\
\hline$\left[1_{-} 0_{+} \infty{ }_{+} a_{-}\right]$ & $\begin{array}{l}x^{-\alpha+\delta-1}(x-1)^{1-\delta} H l\left(\frac{a}{a-1}, \frac{-q+(\beta-1)(\alpha-\delta+1)-[(\gamma+\delta-2)(\alpha-\delta+1)-\gamma \alpha] a}{a-1} ;\right. \\
\begin{array}{c}\left.\alpha-\gamma-\delta+2, \alpha-\delta+1,2-\delta, \alpha+\beta-\gamma-\delta+1 ; \frac{a(x-1)}{(a-1) x}\right) \\
x^{\beta-\gamma-1}(x-1)^{1-\delta}\left(\frac{x-a}{1-a}\right)^{-\alpha-\beta+\gamma+\delta} H l\left(\frac{a}{a-1}, \frac{-q+(\delta \beta-2 \beta+\gamma-\delta+2) a+(\beta-1)(\alpha-\delta+1)}{a-1} ;\right. \\
\left.-\beta+\gamma+1,2-\beta, 2-\delta,-\alpha-\beta+\gamma+\delta+1 ; \frac{a(x-1)}{(a-1) x}\right)\end{array}\end{array}$ \\
\hline$\left.D_{-} a_{+}\right]$ & $\begin{array}{l}x^{-\beta+\delta-1}(x-1)^{1-\delta} H l\left(\frac{a}{a-1}, \frac{-q+(\beta-\delta+1)(\alpha-1)-l(\gamma+\delta-2)(\beta-\delta+1)-\gamma \beta] a}{a-1} ;\right. \\
x^{\alpha-\gamma-1}(x-1)^{1-\delta}\left(\frac{x-a}{1-a}\right)^{-\alpha-\beta+\gamma+\delta} H l\left(\frac{a}{a-1}, \frac{-q+(\delta \alpha-2 \alpha+\gamma-\delta+2) a+(\beta-\delta+1)(\alpha-1)}{a-1} ;\right. \\
\left.2-\alpha,-\alpha+\gamma+1,2-\delta,-\alpha-\beta+\gamma+\delta+1 ; \frac{a(x-1)}{(a-1) x}\right)\end{array}$ \\
\hline
\end{tabular}




\begin{tabular}{|c|c|}
\hline & \\
\hline $\begin{array}{l}{\left[a_{+} 0_{+} \infty_{+} 1_{+}\right]} \\
{\left[a_{+} 0_{-} \infty_{-} 1_{+}\right]} \\
{\left[a_{+} 0_{-} \infty_{+} 1_{-}\right]}\end{array}$ & $\begin{array}{c}\left(\frac{x}{a}\right)^{-\alpha} H l\left(1-a,-q-\alpha[(\alpha-\gamma-\delta+1) a-\alpha-\beta+\gamma+\delta-1] ; \alpha, \alpha-\gamma+1, \alpha+\beta-\gamma-\delta+1, \alpha-\beta+1 ; \frac{x-a}{x}\right) \\
\left(\frac{x}{a}\right)^{-\beta} H l\left(1-a,-q-\beta[(\beta-\gamma-\delta+1) a-\alpha-\beta+\gamma+\delta-1] ; \beta-\gamma+1, \beta, \alpha+\beta-\gamma-\delta+1,-\alpha+\beta+1 ; \frac{x-a}{x}\right) \\
\left(\frac{x}{a}\right)^{-\alpha+\delta-1}\left(\frac{x-1}{a-1}\right)^{1-\delta} H l(1-a,-q+(\alpha-\delta+1)(\alpha+\beta-\gamma-\delta+1)-\alpha(\alpha-\gamma-\delta+1) a ; \\
\left.\quad \alpha-\gamma-\delta+2, \alpha-\delta+1, \alpha+\beta-\gamma-\delta+1, \alpha-\beta+1 ; \frac{x-a}{x}\right) \\
\left(\frac{x}{a}\right)^{-\beta+\delta-1}\left(\frac{x-1}{a-1}\right)^{1-\delta} H l(1-a,-q+(\beta-\delta+1)(\alpha+\beta-\gamma-\delta+1)-\beta(\beta-\gamma-\delta+1) a ; \\
\left.\quad \beta-\delta+1, \beta-\gamma-\delta+2, \alpha+\beta-\gamma-\delta+1,-\alpha+\beta+1 ; \frac{x-a}{x}\right)\end{array}$ \\
\hline $\begin{array}{l}{\left[a_{+} 0_{+} 1_{+}\right]\left[\infty_{+}\right]} \\
{\left[a_{+} 0_{-} 1_{+}\right]\left[\infty_{-}\right]} \\
{\left[a_{+} 0_{+} 1_{-}\right]\left[\infty_{-}\right]} \\
{\left[a_{+} 0_{-} 1_{-}\right]\left[\infty_{+}\right]}\end{array}$ & $\begin{array}{l}H l\left(\frac{a-1}{a}, \frac{-q+\beta \alpha a}{a} ; \alpha, \beta, \alpha+\beta-\gamma-\delta+1, \gamma ; \frac{a-x}{a}\right) \\
\left(\frac{x}{a}\right)^{1-\gamma} H l\left(\frac{a-1}{a}, \frac{-q+[\beta \alpha-(\gamma-1)(\alpha+\beta-\gamma-\delta+1)] a+(\gamma-1)(\alpha+\beta-\gamma-\delta+1)}{a} ; \beta-\gamma+1, \alpha-\gamma+1, \alpha+\beta-\gamma-\delta+1,2-\gamma ; \frac{a-x}{a}\right) \\
\left(\frac{x-1}{a-1}\right)^{1-\delta} H l\left(\frac{a-1}{a}, \frac{-q+[\beta \alpha-(\delta-1)(\alpha+\beta-\gamma-\delta+1)] a}{a} ; \beta-\delta+1, \alpha-\delta+1, \alpha+\beta-\gamma-\delta+1, \gamma ; \frac{a-x}{a}\right) \\
\left(\frac{x}{a}\right)^{1-\gamma}\left(\frac{x-1}{a-1}\right)^{1-\delta} \operatorname{Hl}\left(\frac{a-1}{a}, \frac{-q+[\beta \alpha-(\gamma+\delta-2)(\alpha+\beta-\gamma-\delta+1)] a+(\gamma-1)(\alpha+\beta-\gamma-\delta+1)}{a} ;\right. \\
\left.\alpha-\gamma-\delta+2, \beta-\gamma-\delta+2, \alpha+\beta-\gamma-\delta+1,2-\gamma ; \frac{a-x}{a}\right)\end{array}$ \\
\hline $\begin{array}{l}{\left[a_{+} 0_{+}\right]\left[1_{+} \infty_{+}\right]} \\
{\left[a_{+} 0_{+}\right]\left[1_{-} \infty_{-}\right]} \\
{\left[a_{+} 0_{-}\right]\left[1_{-} \infty_{+}\right]} \\
{\left[a_{+} 0_{-}\right]\left[1_{+} \infty_{-}\right]}\end{array}$ & $\begin{array}{l}\left(\frac{x-1}{a-1}\right)^{-\alpha} \operatorname{Hl}\left(a, q+\alpha(\alpha-\gamma-\delta+1) a ; \alpha, \alpha-\delta+1, \alpha+\beta-\gamma-\delta+1, \alpha-\beta+1 ; \frac{x-a}{x-1}\right) \\
\left(\frac{x-1}{a-1}\right)^{-\beta} H l\left(a, q+\beta(\beta-\gamma-\delta+1) a ; \beta-\delta+1, \beta, \alpha+\beta-\gamma-\delta+1,-\alpha+\beta+1 ; \frac{x-a}{x-1}\right) \\
\left(\frac{x}{a}\right)^{1-\gamma}\left(\frac{x-1}{a-1}\right)^{-\alpha+\gamma-1} \operatorname{Hl}(a, q+\alpha(\alpha-\gamma-\delta+1) a-(\gamma-1)(\alpha+\beta-\gamma-\delta+1) ; \\
\left.\quad \alpha-\gamma-\delta+2, \alpha-\gamma+1, \alpha+\beta-\gamma-\delta+1, \alpha-\beta+1 ; \frac{x-a}{x-1}\right) \\
\left(\frac{x}{a}\right)^{1-\gamma}\left(\frac{x-1}{a-1}\right)^{-\beta+\gamma-1} \operatorname{Hl}(a, q+\beta(\beta-\gamma-\delta+1) a-(\gamma-1)(\alpha+\beta-\gamma-\delta+1) ; \\
\left.\beta-\gamma+1, \beta-\gamma-\delta+2, \alpha+\beta-\gamma-\delta+1,-\alpha+\beta+1 ; \frac{x-a}{x-1}\right)\end{array}$ \\
\hline $\begin{array}{l}{\left[a_{+} 0_{+}\right]\left[1_{+}\right]\left[\infty_{+}\right]} \\
{\left[a_{+} 0_{+}\right]\left[1_{-}\right]\left[\infty_{-}\right]} \\
{\left[a_{+} 0_{-}\right]\left[1_{+}\right]\left[\infty_{-}\right]} \\
{\left[a_{+} 0_{-}\right]\left[1_{-}\right]\left[\infty_{+}\right]}\end{array}$ & $\begin{array}{l}H l\left(\frac{a}{a-1}, \frac{-q+\beta \alpha a}{a-1} ; \alpha, \beta, \alpha+\beta-\gamma-\delta+1, \delta ; \frac{a-x}{a-1}\right) \\
\left(\frac{x-1}{a-1}\right)^{1-\delta} H l\left(\frac{a}{a-1}, \frac{-q+[\beta \alpha-(\delta-1)(\alpha+\beta-\gamma-\delta+1)] a}{a-1} ; \beta-\delta+1, \alpha-\delta+1, \alpha+\beta-\gamma-\delta+1,2-\delta ; \frac{a-x}{a-1}\right) \\
\left(\frac{x}{a}\right)^{1-\gamma} H l\left(\frac{a}{a-1}, \frac{-q+[\beta \alpha-(\gamma-1)(\alpha+\beta-\gamma-\delta+1)] a+(\gamma-1)(\alpha+\beta-\gamma-\delta+1)}{a-1} ; \beta-\gamma+1, \alpha-\gamma+1, \alpha+\beta-\gamma-\delta+1, \delta ; \frac{a-x}{a-1}\right) \\
\left(\frac{x}{a}\right)^{1-\gamma}\left(\frac{x-1}{a-1}\right)^{1-\delta} H l\left(\frac{a}{a-1}, \frac{-q+[\beta \alpha-(\gamma+\delta-2)(\alpha+\beta-\gamma-\delta+1)] a+(\gamma-1)(\alpha+\beta-\gamma-\delta+1)}{a-1} ;\right. \\
\left.\alpha-\gamma-\delta+2, \beta-\gamma-\delta+2, \alpha+\beta-\gamma-\delta+1,2-\delta ; \frac{a-x}{a-1}\right)\end{array}$ \\
\hline
\end{tabular}




\begin{tabular}{|c|c|}
\hline $\left.\begin{array}{l}+ \\
+ \\
+ \\
+ \\
-]\end{array}\right]$ & $\begin{array}{l}\left(\frac{x-1}{a-1}\right)^{-\alpha} H l\left(\frac{1}{a}, \frac{q+\alpha(\alpha-\gamma-\delta+1) a}{a} ; \alpha, \alpha-\delta+1, \alpha+\beta-\gamma-\delta+1, \gamma ; \frac{x-a}{a(x-1)}\right) \\
\left(\frac{x}{a}\right)^{1-\gamma}\left(\frac{x-1}{a-1}\right)^{-\alpha+\gamma-1} \operatorname{Hl}\left(\frac{1}{a}, \frac{q+\alpha(\alpha-\gamma-\delta+1) a-(\gamma-1)(\alpha+\beta-\gamma-\delta+1)}{a} ; \alpha-\gamma-\delta+2, \alpha-\gamma+1, \alpha+\beta-\gamma-\delta+1,2-\gamma ; \frac{x-a}{a(x-1)}\right) \\
\left(\frac{x-1}{a-1}\right)^{-\beta} H l\left(\frac{1}{a}, \frac{q+\beta(\beta-\gamma-\delta+1) a}{a} ; \beta-\delta+1, \beta, \alpha+\beta-\gamma-\delta+1, \gamma ; \frac{x-a}{a(x-1)}\right) \\
\left(\frac{x}{a}\right)^{1-\gamma}\left(\frac{x-1}{a-1}\right)^{-\beta+\gamma-1} \operatorname{Hl}\left(\frac{1}{a}, \frac{q+\beta(\beta-\gamma-\delta+1) a-(\gamma-1)(\alpha+\beta-\gamma-\delta+1)}{a} ; \beta-\gamma+1, \beta-\gamma-\delta+2, \alpha+\beta-\gamma-\delta+1,2-\gamma ; \frac{x-a}{a(x-1)}\right)\end{array}$ \\
\hline$\left.\infty_{-}\right]\left[1_{+}\right]$ & $\begin{array}{l}\left(\frac{x}{a}\right)^{-\alpha} H l\left(\frac{1}{1-a}, \frac{q+\alpha[(\alpha-\gamma-\delta+1) a-\alpha-\beta+\gamma+\delta-1]}{a-1} ; \alpha, \alpha-\gamma+1, \alpha+\beta-\gamma-\delta+1, \delta ; \frac{a-x}{(a-1) x}\right) \\
\left(\frac{x}{a}\right)^{-\alpha+\delta-1}\left(\frac{x-1}{a-1}\right)^{1-\delta} H l\left(\frac{1}{1-a}, \frac{q+\alpha(\alpha-\gamma-\delta+1) a-(\alpha-\delta+1)(\alpha+\beta-\gamma-\delta+1)}{a-1} ;\right. \\
\left(\frac{x}{a}\right)^{-\beta} H l\left(\frac{1}{1-a}, \frac{q+\beta[(\beta-\gamma-\delta+1) a-\alpha-\beta+\gamma+\delta-1]}{a-1} ; \beta-\gamma+1, \beta, \alpha+\beta-\gamma-\delta+1, \delta ; \frac{a-x}{(a-1) x}\right) \\
\left(\frac{x}{a}\right)^{-\beta+\delta-1}\left(\frac{x-1}{a-1}\right)^{1-\delta} H l\left(\frac{1}{1-a}, \frac{q+\beta(\beta-\gamma-\delta+1) a-(\beta-\delta+1)(\alpha+\beta-\gamma-\delta+1)}{a-1} ;\right. \\
\left.\beta-\delta+1, \beta-\gamma-\delta+2, \alpha+\beta-\gamma-\delta+1,2-\delta ; \frac{a-x}{(a-1) x}\right)\end{array}$ \\
\hline & $\overline{c t} 1-\epsilon)$ \\
\hline $\begin{array}{c}+] \\
\left.1_{+}\right]\end{array}$ & 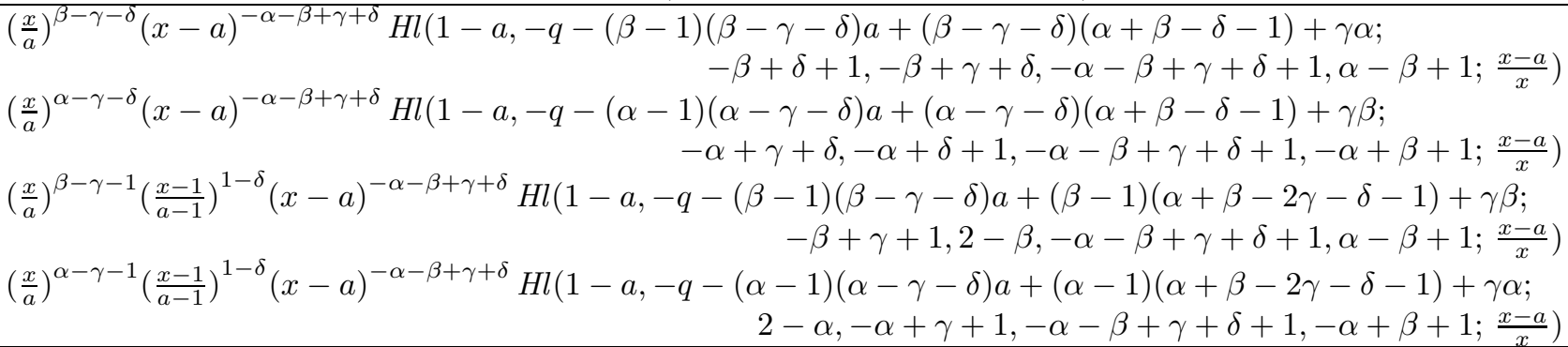 \\
\hline
\end{tabular}




\begin{tabular}{|c|c|}
\hline $\begin{array}{l}{\left[a_{-} 0_{+} 1_{+}\right]\left[\infty_{-}\right]} \\
{\left[a_{-} 0_{-} 1_{+}\right]\left[\infty_{+}\right]}\end{array}$ & 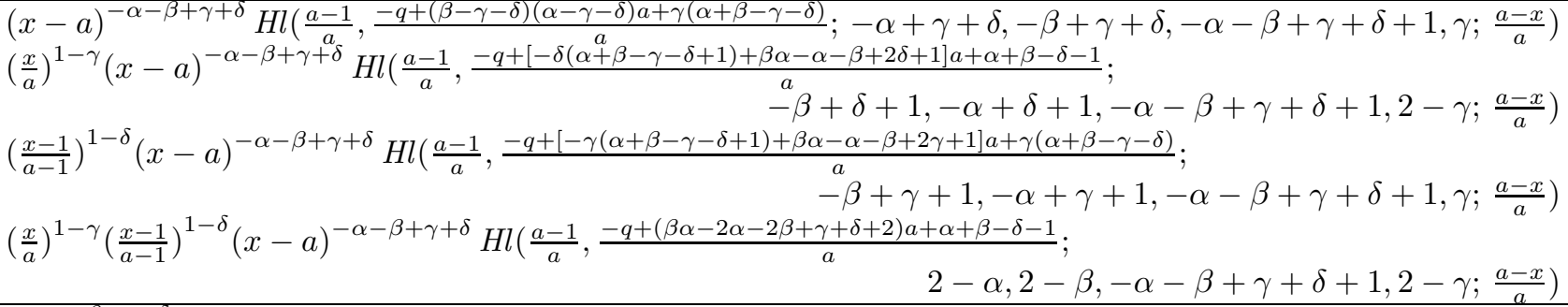 \\
\hline $\begin{array}{l}{\left[a_{-} 0_{+}\right]\left[1_{-} \infty_{+}\right]} \\
{\left[a_{-} 0_{+}\right]\left[1_{+} \infty_{-}\right]} \\
{\left[a_{-} 0_{-}\right]\left[1_{+} \infty_{+}\right]}\end{array}$ & 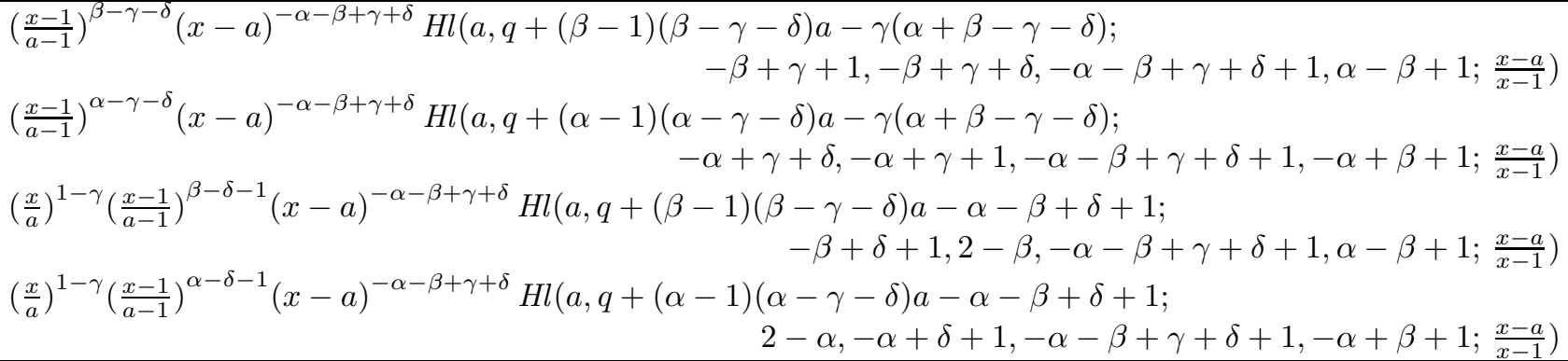 \\
\hline $\begin{array}{l}{\left[a_{-} 0_{+}\right]\left[1_{+}\right]\left[\infty_{-}\right]} \\
{\left[a_{-} 0_{+}\right]\left[1_{-}\right]\left[\infty_{+}\right]}\end{array}$ & 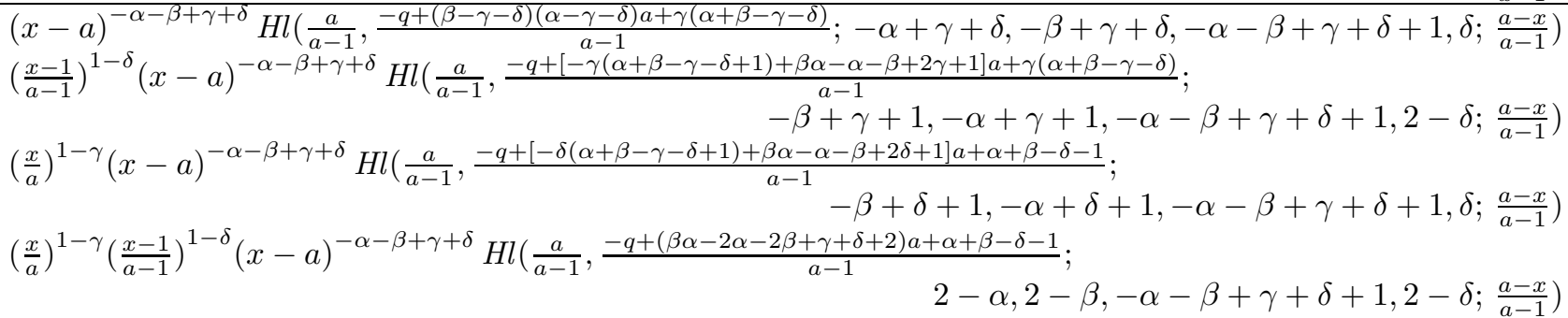 \\
\hline
\end{tabular}




\begin{tabular}{|c|c|}
\hline$\left[a_{-} 0\right.$ & $\left(\frac{x-1}{a-1}\right)^{\beta-\gamma-\delta}(x-a)^{-\alpha-\beta+\gamma+\delta} H l\left(\frac{1}{a}, \frac{q+(\beta-1)(\beta-\gamma-\delta) a-\gamma(\alpha+\beta-\gamma-\delta)}{a}-\beta+\gamma+1,-\beta+\gamma+\delta,-\alpha-\beta+\gamma+\delta+1, \gamma ; \frac{x-a}{a(x-1)}\right)$ \\
\hline$\left[a_{-} 0_{-} 1_{+} \infty_{+}\right]$ & 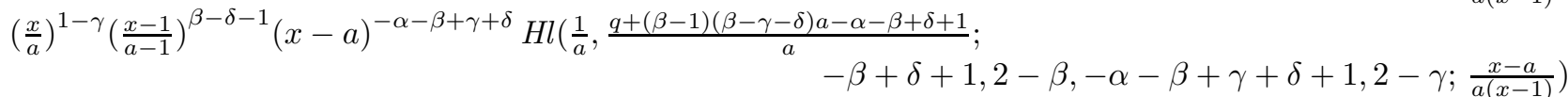 \\
\hline$\left[a_{-} 0_{+} 1_{+} \infty_{-}\right]$ & $\left(\frac{x-1}{a-1}\right)^{\alpha-\gamma-\delta}(x-a)^{-\alpha-\beta+\gamma+\delta} H l\left(\frac{1}{a}, \frac{q+(\alpha-1)(\alpha-\gamma-\delta) a-\gamma(\alpha+\beta-\gamma-\delta)}{a} ;-\alpha+\gamma+\delta,-\alpha+\gamma+1,-\alpha-\beta+\gamma+\delta+1, \gamma ; \frac{x-a}{a(x-1)}\right)$ \\
\hline$\left[a_{-} 0\right.$ & $\left(\frac{x}{a}\right)^{1-\gamma}\left(\frac{x-1}{a-1}\right)^{\alpha-\delta-1}(x-a)^{-\alpha-\beta+\gamma+\delta} H l\left(\frac{1}{a}, \frac{q+(\alpha-1)(\alpha-\gamma-\delta) a-\alpha-\beta+\delta+1}{a} ;\right.$ \\
\hline$\left[a_{-} 0_{-} \infty_{+}\right]\left[1_{+}\right]$ & $\left(\frac{x}{a}\right)^{\beta-\gamma-\delta}(x-a)^{-\alpha-\beta+\gamma+\delta} H l\left(\frac{1}{1-a}, \frac{q+(\beta-1)(\beta-\gamma-\delta) a-(\beta-\gamma-\delta)(\alpha+\beta-\delta-1)-\gamma \alpha}{a-1} ;\right.$ \\
\hline$\left[a_{-} 0_{+} \infty\right.$ & $\left(\frac{x}{a}\right)^{\beta-\gamma-1}\left(\frac{x-1}{a-1}\right)^{1-\delta}(x-a)^{-\alpha-\beta+\gamma+\delta} H l\left(\frac{1}{1-a}, \frac{q+(\beta-1)(\beta-\gamma-\delta) a-(\beta-1)(\alpha+\beta-2 \gamma-\delta-1)-\gamma \beta}{a-1} ;\right.$ \\
\hline$\left[a_{-} 0_{+} \infty_{-}\right]\left[1_{+}\right]$ & 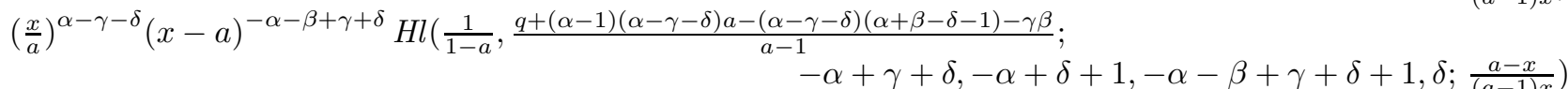 \\
\hline$\left[a_{-} 0_{-}\right.$ & $\begin{array}{c}-1)(\alpha-\gamma-\delta) a-(\alpha-1)(\alpha+\beta-2 \gamma-\delta-1)-\gamma \alpha \\
2-\alpha,-\alpha+\gamma+1,-\alpha-\beta+\gamma\end{array}$ \\
\hline \multicolumn{2}{|r|}{ Frobenius solution $\# 1$ at $x=\infty($ characteristic exponent $\alpha)$} \\
\hline$\left[\infty_{+} 0_{+}\right]\left[1_{+}\right]\left[a_{+}\right]$ & $\overline{-\alpha} H l\left(\frac{1}{a}, \underline{q+\alpha}\right.$ \\
\hline +] & $\left.\frac{1-1)}{\alpha} ; \alpha-\gamma-\delta+2, \alpha-\delta+1, \alpha-\beta+1,2-\delta ; \frac{1}{x}\right)$ \\
\hline & $\left.\frac{-\delta) \alpha}{2} ;-\beta+\delta+1,-\beta+\gamma+\delta, \alpha-\beta+1, \delta ; \frac{1}{x}\right)$ \\
\hline$\left.\infty_{+} 0_{+}\right]\left[1_{-}\right]\left[a_{-}\right]$ & $\left.\frac{\beta-\gamma-\delta) a-\alpha+\delta-1]}{a} ;-\beta+\gamma+1,2-\beta, \alpha-\beta+1,2-\delta ; \frac{1}{x}\right)$ \\
\hline
\end{tabular}




\begin{tabular}{|c|c|}
\hline$\left[\infty_{+} 0_{+} 1_{-}\right]\left[a_{-}\right]$ & 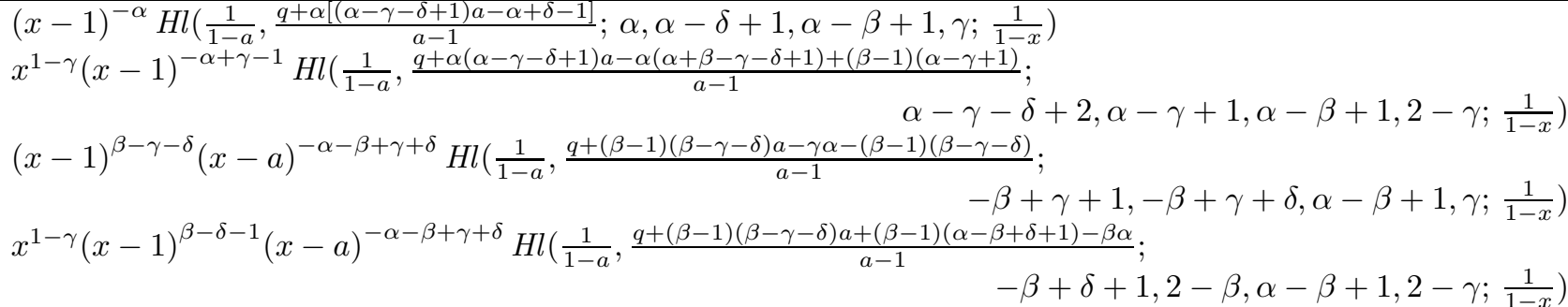 \\
\hline $\begin{array}{l}{\left[\infty_{+} 0_{+}\right]\left[1_{+} a_{+}\right]} \\
{\left[\infty_{+} 0_{-}\right]\left[1_{+} a_{-}\right]} \\
{\left[\infty_{+} 0_{-}\right]\left[1_{-} a_{+}\right]} \\
{\left[\infty_{+} 0_{+}\right]\left[1_{-} a_{-}\right]}\end{array}$ & $\begin{array}{l}\left.\begin{array}{l}x^{-\alpha} H l(a, q+\alpha[(\alpha-\gamma-\delta+1) a-\beta+\delta] ; \alpha, \alpha-\gamma+1, \alpha-\beta+1, \alpha+\beta-\gamma-\delta+1 ; \\
x\end{array}\right) \\
x^{\beta-\gamma-\delta}(x-a)^{-\alpha-\beta+\gamma+\delta} \operatorname{Hl}(a, q+(\beta-1)(\beta-\gamma-\delta) a-(\beta-\delta) \alpha ; \\
\left.\quad-\beta+\delta+1,-\beta+\gamma+\delta, \alpha-\beta+1,-\alpha-\beta+\gamma+\delta+1 ; \frac{a}{x}\right) \\
x^{-\alpha+\delta-1}(x-1)^{1-\delta} H l(a, q+\alpha(\alpha-\gamma-\delta+1) a-(\beta-1)(\alpha-\delta+1) ; \\
\left.\alpha-\gamma-\delta+2, \alpha-\delta+1, \alpha-\beta+1, \alpha+\beta-\gamma-\delta+1 ; \frac{a}{x}\right) \\
x^{\beta-\gamma-1}(x-1)^{1-\delta}(x-a)^{-\alpha-\beta+\gamma+\delta} \operatorname{Hl}(a, q+(\beta-1)[(\beta-\gamma-\delta) a-\alpha+\delta-1] ; \\
\left.-\beta+\gamma+1,2-\beta, \alpha-\beta+1,-\alpha-\beta+\gamma+\delta+1 ; \frac{a}{x}\right)\end{array}$ \\
\hline $\begin{array}{l}\left.{ }_{+} a_{+}\right] \\
\left.+a_{-}\right] \\
\left.-a_{-}\right] \\
\left.-a_{+}\right]\end{array}$ & $\begin{array}{l}(x-a)^{-\alpha} H l\left(\frac{a}{a-1}, \frac{-q-\alpha[(\beta-\gamma-\delta) a-\beta+\delta]}{a-1} ; \alpha,-\beta+\gamma+\delta, \alpha-\beta+1, \gamma ; \frac{a}{a-x}\right) \\
x^{1-\gamma}(x-a)^{-\alpha+\gamma-1} \operatorname{Hl}\left(\frac{a}{a-1}, \frac{-q+(\beta-\delta) \alpha-[(\beta-1)(\alpha-\gamma+1)-\delta \alpha] a}{a-1} ;-\beta+\delta+1, \alpha-\gamma+1, \alpha-\beta+1,2-\gamma ; \frac{a}{a-x}\right) \\
(x-1)^{1-\delta}(x-a)^{-\alpha+\delta-1} \operatorname{Hl}\left(\frac{a}{a-1}, \frac{-q+(\beta-1)(\alpha-\delta+1)-[(\beta-1)(\alpha-\delta+1)-\gamma \alpha] a}{a-1} ;-\beta+\gamma+1, \alpha-\delta+1, \alpha-\beta+1, \gamma ; \frac{a}{a-x}\right) \\
x^{1-\gamma}(x-1)^{1-\delta}(x-a)^{-\alpha+\gamma+\delta-2} \operatorname{Hl}\left(\frac{a}{a-1}, \frac{-q+(\beta-1)(\alpha-\delta+1)-[(\beta-1)(2 \alpha-\gamma-\delta+2)-\beta \alpha] a}{a-1} ;\right. \\
\left.\alpha-\gamma-\delta+2,2-\beta, \alpha-\beta+1,2-\gamma ; \frac{a}{a-x}\right)\end{array}$ \\
\hline $\begin{array}{l}\left.l_{+} 1_{+}\right] \\
\left.u_{-} 1_{-}\right]\end{array}$ & $\begin{array}{r}(x-1)^{-\alpha} H l\left(1-a,-q-\alpha[(\alpha-\gamma-\delta+1) a-\alpha+\delta-1] ; \alpha, \alpha-\delta+1, \alpha-\beta+1, \alpha+\beta-\gamma-\delta+1 ; \frac{a-1}{x-1}\right) \\
(x-1)^{\beta-\gamma-\delta}(x-a)^{-\alpha-\beta+\gamma+\delta} H l(1-a,-q-(\beta-1)(\beta-\gamma-\delta) a+\gamma \alpha+(\beta-1)(\beta-\gamma-\delta) ; \\
\left.-\beta+\gamma+1,-\beta+\gamma+\delta, \alpha-\beta+1,-\alpha-\beta+\gamma+\delta+1 ; \frac{a-1}{x-1}\right) \\
x^{1-\gamma}(x-1)^{-\alpha+\gamma-1} H l(1-a,-q-\alpha(\alpha-\gamma-\delta+1) a+\alpha(\alpha+\beta-\gamma-\delta+1)-(\beta-1)(\alpha-\gamma+1) ; \\
\left.\alpha-\gamma-\delta+2, \alpha-\gamma+1, \alpha-\beta+1, \alpha+\beta-\gamma-\delta+1 ; \frac{a-1}{x-1}\right) \\
x^{1-\gamma}(x-1)^{\beta-\delta-1}(x-a)^{-\alpha-\beta+\gamma+\delta} H l(1-a,-q-(\beta-1)(\beta-\gamma-\delta) a-(\beta-1)(\alpha-\beta+\delta+1)+\beta \alpha ; \\
\left.-\beta+\delta+1,2-\beta, \alpha-\beta+1,-\alpha-\beta+\gamma+\delta+1 ; \frac{a-1}{x-1}\right)\end{array}$ \\
\hline
\end{tabular}




\begin{tabular}{|c|c|}
\hline $\left.\begin{array}{l}+]\left[1_{+}\right] \\
-]\left[1_{-}\right] \\
-]\left[1_{+}\right] \\
+\end{array}\right]\left[1_{-}\right]$ & $\begin{array}{l}-a)^{-\alpha} \operatorname{Hl}\left(\frac{a-1}{a}, \frac{-q-\alpha[(\rho-\gamma-\gamma) a-p+o]}{a} ; \alpha,-\beta+\gamma+\delta, \alpha-\beta+1, \delta ; \frac{1-a}{x-a}\right) \\
-1)^{1-\delta}(x-a)^{-\alpha+\delta-1} \operatorname{Hl}\left(\frac{a-1}{a}, \frac{-q+(\beta-1)(\alpha-\delta+1)-[(\beta-1)(\alpha-\delta+1)-\gamma \alpha] a}{a} ;-\beta+\gamma+1, \alpha-\delta+1, \alpha-\beta+1,2-\delta ; \frac{1-a}{x-a}\right) \\
-\gamma(x-a)^{-\alpha+\gamma-1} \operatorname{Hl}\left(\frac{a-1}{a}, \frac{-q+(\beta-\delta) \alpha-(\beta \alpha-\delta \alpha-\alpha-\gamma \beta+\beta+\gamma-1) a}{a} ;-\beta+\delta+1, \alpha-\gamma+1, \alpha-\beta+1, \delta ; \frac{1-a}{x-a}\right) \\
-\gamma(x-1)^{1-\delta}(x-a)^{-\alpha+\gamma+\delta-2} \operatorname{Hl}\left(\frac{a-1}{a}, \frac{-q+(\beta-1)(\alpha-\delta+1)-[(\beta-1)(2 \alpha-\gamma-\delta+2)-\beta \alpha] a}{a} ;\right. \\
\left.\alpha-\gamma-\delta+2,2-\beta, \alpha-\beta+1,2-\delta ; \frac{1-a}{x-a}\right)\end{array}$ \\
\hline \multicolumn{2}{|r|}{ Frobenius solution $\# 2$ at $x=\infty($ characteristic exponent $\beta)$} \\
\hline $\begin{array}{l}]\left[a_{+}\right] \\
]\left[a_{+}\right] \\
]\left[a_{-}\right] \\
]\left[a_{-}\right]\end{array}$ & 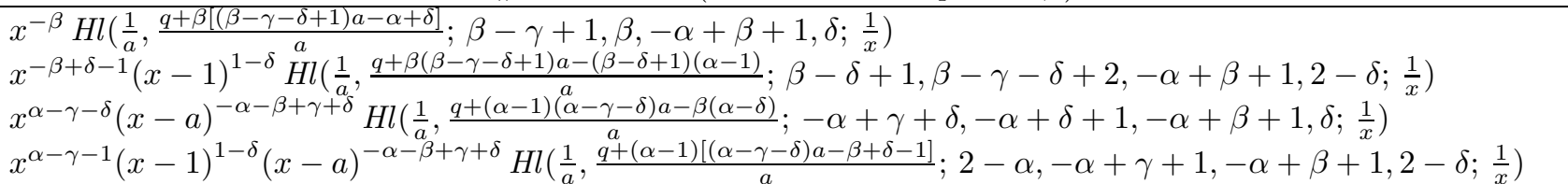 \\
\hline $\begin{array}{l}{[\infty} \\
{[\infty}\end{array}$ & $\begin{array}{l}(x-1)^{-\beta} H l\left(\frac{1}{1-a}, \frac{q+\beta[(\beta-\gamma-\delta+1) a-\beta+\delta-1]}{a-\beta} ; \beta-\delta+1, \beta,-\alpha+\beta+1, \gamma ; \frac{1}{1-x}\right) \\
x^{1-\gamma}(x-1)^{-\beta+\gamma-1} \operatorname{Hl}\left(\frac{1}{1-a}, \frac{q+\beta(\beta-\gamma-\delta+1) a-\beta(\alpha+\beta-\gamma-\delta+1)+(\beta-\gamma+1)(\alpha-1)}{a-1} ;\right. \\
(x-1)^{\alpha-\gamma-\delta}(x-a)^{-\alpha-\beta+\gamma+\delta} H l\left(\frac{1}{1-a}, \frac{q+(\alpha-1)(\alpha-\gamma-\delta) a-(\alpha-1)(\alpha-\gamma-\delta)-\gamma \beta}{a-1} ;\right.\end{array}$ \\
\hline $\begin{array}{l}\left.+a_{+}\right] \\
\left.a_{-}\right] \\
\left.a_{+}\right]\end{array}$ & 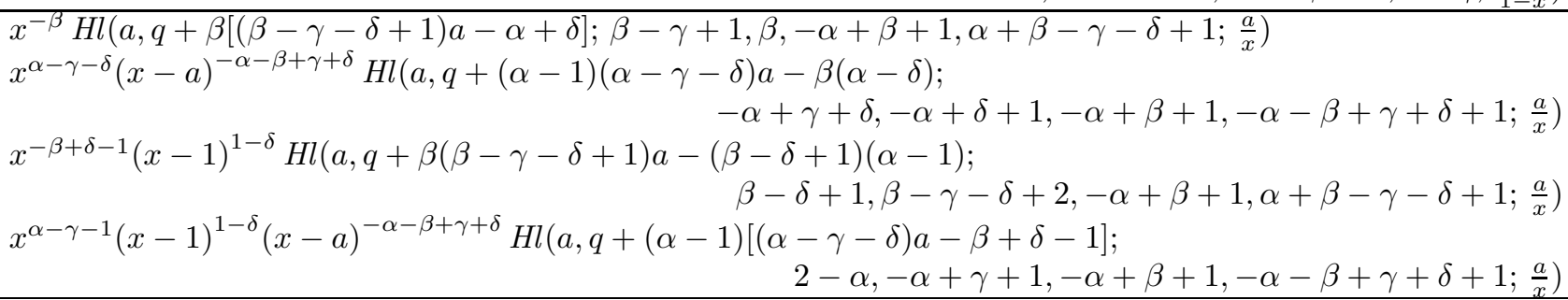 \\
\hline
\end{tabular}




\begin{tabular}{|c|c|}
\hline $\begin{array}{r}\left.{ }_{+} a_{+}\right] \\
\left.-a_{+}\right]\end{array}$ & $\begin{array}{l}(x-a)^{-\beta} H l\left(\frac{a}{a-1}, \frac{-q-\beta[(\alpha-\gamma-\delta) a-\alpha+\delta]}{a-1} ;-\alpha+\gamma+\delta, \beta,-\alpha+\beta+1, \gamma ; \frac{a}{a-x}\right) \\
x^{1-\gamma}(x-a)^{-\beta+\gamma-1} H l\left(\frac{a}{a-1}, \frac{-q+\beta(\alpha-\delta)-[(\beta-\gamma+1)(\alpha-1)-\delta \beta] a}{a-1} ; \beta-\gamma+1,-\alpha+\delta+1,-\alpha+\beta+1,2-\gamma ; \frac{a}{a-x}\right) \\
(x-1)^{1-\delta}(x-a)^{-\beta+\delta-1} H l\left(\frac{a}{a-1}, \frac{-q+(\beta-\delta+1)(\alpha-1)-[(\beta-\delta+1)(\alpha-1)-\gamma \beta] a}{a-1} ; \beta-\delta+1,-\alpha+\gamma+1,-\alpha+\beta+1, \gamma ; \frac{a}{a-x}\right) \\
\begin{array}{r}x^{1-\gamma}(x-1)^{1-\delta}(x-a)^{-\beta+\gamma+\delta-2} H l\left(\frac{a}{a-1}, \frac{-q+(\beta-\delta+1)(\alpha-1)-[(2 \beta-\gamma-\delta+2)(\alpha-1)-\beta \alpha] a}{a-1} ;\right. \\
\left.2-\alpha, \beta-\gamma-\delta+2,-\alpha+\beta+1,2-\gamma ; \frac{a}{a-x}\right)\end{array}\end{array}$ \\
\hline$[\infty$ & $\begin{array}{r}(x-1)^{-\beta} H l(1-a,-q-\beta[(\beta-\gamma-\delta+1) a-\beta+\delta-1] ; \beta-\delta+1, \beta,-\alpha+\beta \\
(x-1)^{\alpha-\gamma-\delta}(x-a)^{-\alpha-\beta+\gamma+\delta} H l(1-a,-q-(\alpha-1)(\alpha-\gamma-\delta) a+(\alpha-1)(\alpha \\
-\alpha+\gamma+\delta,-\alpha+\gamma+1,-\alpha+ \\
x^{1-\gamma}(x-1)^{-\beta+\gamma-1} H l(1-a,-q-\beta(\beta-\gamma-\delta+1) a+\beta(\alpha+\beta-\gamma-\delta+1)- \\
\beta-\gamma+1, \beta-\gamma-\delta+2,-\alpha- \\
x^{1-\gamma}(x-1)^{\alpha-\delta-1}(x-a)^{-\alpha-\beta+\gamma+\delta} H l(1-a,-q-(\alpha-1)(\alpha-\gamma-\delta) a+(\alpha- \\
2-\alpha,-\alpha+\delta+1,-\alpha+\end{array}$ \\
\hline $\begin{array}{l}{\left[\infty_{-} 0_{+}\right.} \\
{\left[\infty_{-} 0_{+}\right.}\end{array}$ & $\begin{array}{l}(x-a)^{-\beta} H l\left(\frac{a-1}{a}, \frac{-q-\beta \mid(\alpha-\gamma-\delta) a-\alpha+\delta]}{a} ;-\alpha+\gamma+\delta, \beta,-\alpha+\beta+1, \delta ; \frac{1-a}{x-a}\right) \\
(x-1)^{1-\delta}(x-a)^{-\beta+\delta-1} \operatorname{Hl}\left(\frac{a-1}{a}, \frac{-q+(\beta-\delta+1)(\alpha-1)-[(\beta-\delta+1)(\alpha-1)-\gamma \beta] a}{a} ;\right. \\
\left.\beta-\delta+1,-\alpha+\gamma+1,-\alpha+\beta+1,2-\delta ; \frac{1-a}{x-a}\right) \\
x^{1-\gamma}(x-a)^{-\beta+\gamma-1} H l\left(\frac{a-1}{a}, \frac{-q+\beta(\alpha-\delta)-(\beta \alpha-\gamma \alpha+\alpha-\delta \beta-\beta+\gamma-1) a}{a} ; \beta-\gamma+1,-\alpha+\delta+1,-\alpha+\beta+1, \delta ; \frac{1-a}{x-a}\right) \\
x^{1-\gamma}(x-1)^{1-\delta}(x-a)^{-\beta+\gamma+\delta-2} \operatorname{Hl}\left(\frac{a-1}{a}, \frac{-q+(\beta-\delta+1)(\alpha-1)-[(2 \beta-\gamma-\delta+2)(\alpha-1)-\beta \alpha] a}{a} ;\right. \\
\left.2-\alpha, \beta-\gamma-\delta+2,-\alpha+\beta+1,2-\delta ; \frac{1-a}{x-a}\right)\end{array}$ \\
\hline
\end{tabular}


The tabulated solutions are partitioned, respectively, into 6 sets of 4 and 8 sets of 24. These are sets of equivalent expressions for the 6 (resp. 8) Frobenius solutions, each defined in a neighborhood of one of the 3 (resp. 4) singular points. They are further partitioned by the Möbius transformation performed on the independent variable $x$, which is in one-to-one correspondence with the unannotated cycle representation of $g$. The Möbius transformations appearing in Tables 1 and 2 belong to the Kummer group (3.1) and Heun group (3.2), respectively. Although the Heun group has order 24, only 6 transformed values $a^{\prime}$ of the Heun parameter $a$ appear in Table 2, in all. This agrees with the remarks made in Example 3.5 on the size of generic $a$-orbits. If $a \in\left\{-1, \frac{1}{2}, 2\right\}$ or $a \in\left\{\frac{1}{2} \pm \mathrm{i} \frac{\sqrt{3}}{2}\right\}$, the number of distinct values $a^{\prime}$ appearing in the 192 solutions will be smaller: 3 and 2, respectively.

The expressions in Table 1 are the 24 expressions of Kummer [13], which can be found in many modern works $11,5,6,9,17,18$. But the explicit indexing by $g \in \mathcal{D}_{3}$ is unusual (cf. [18]). Nearly all the 192 expressions in Table 2 have never appeared in print before, and the indexing by $g \in \mathcal{D}_{4}$ is entirely new. The most important Frobenius solution in each of Tables 1 and 2 is the first, since it provides the possible transformations of ${ }_{2} F_{1}$, resp., $\mathrm{Hl}$. In the sense of Corollary 4.6, the transformation group of ${ }_{2} F_{1}$ is the order- 4 group $G_{\text {asym }, 0}(3) \cong \mathcal{D}_{2}$ (i.e., the Klein 4-group $\mathbb{Z}_{2} \times \mathbb{Z}_{2}$ ), and that of $\mathrm{Hl}$ is the order-24 group $G_{\text {asym }, 0}(4) \cong \mathcal{D}_{3} \cong S_{4}$.

The transformations of ${ }_{2} F_{1}$ labeled by $\left[1_{-}\right]\left[\infty_{-}\right],\left[1_{+} \infty_{+}\right]$in Table 1 are classical transformations due to Euler and Pfaff [2, 18. This explains why their counterparts, for any $n$, were called generalized Euler and Pfaff transformations in \$4. It is well known that Euler's transformation of ${ }_{2} F_{1}$ can be obtained by iterating Pfaff's 2 , $\S 2.2]$, but the iteration includes an interchange of the exponents $a, b$ at infinity, which induces a twisted composition in $\mathcal{D}_{2}$, or a composition in the order- 8 dihedral group $\mathcal{B}_{2}$, rather than an ordinary composition in $\mathcal{D}_{2}$. This generalizes to any $n \geqslant 3$. The transformations labeled by $\left[1_{-}\right]\left[\infty_{-}\right],\left[1_{+} \infty_{+}\right]$in Table 2 , namely

$$
\begin{aligned}
H l(a, q ; \alpha, \beta, \gamma, \delta ; x) \\
\quad=(1-x)^{1-\delta} H l(a, q-(\delta-1) \gamma a ; \beta-\delta+1, \alpha-\delta+1, \gamma, 2-\delta ; x) \\
\quad=(1-x)^{-\alpha} \operatorname{Hl}\left(\frac{a}{a-1}, \frac{-q+\gamma \alpha a}{a-1} ; \alpha, \alpha-\delta+1, \gamma, \alpha-\beta+1 ; \frac{x}{x-1}\right),
\end{aligned}
$$

are the generalizations of the Euler and Pfaff transformations to the Heun equation. Snow [25, p. 95] obtained the former transformation of $\mathrm{Hl}$ from the latter by what amounts to a twisted composition in $\mathcal{D}_{3}$, or a composition in $\mathcal{B}_{3}$. Transformed values of the accessory parameter $q$ are painfully difficult to compute by hand, so for hand computation, any shortcut is valuable.

The group structure of $\mathcal{D}_{3} \cong S_{4}$ supplies many such shortcuts, as does the structure of $\mathcal{B}_{3}$, the order- 48 extended transformation group of $\mathrm{Hl}$ that includes the interchange of the exponents $\alpha, \beta$ at infinity. (The 24 equivalent expressions for $\mathrm{Hl}$ grow to 48 if this interchange is applied.) Useful results on $\mathcal{B}_{n}$, such as the number of its conjugacy classes and the maximum order of its elements, are readily available [3]. For example, the maximum order of the elements of $\mathcal{B}_{3}$ is 6 , though the maximum order of the elements of $\mathcal{D}_{3} \cong S_{4}$ is 4 . By examination, one of the elements of order 6 is $\left[1_{+} a_{-} \infty_{+}\right] \in \mathcal{B}_{3} \backslash \mathcal{D}_{3}$. In

$$
\left[1_{+} a_{-} \infty_{+}\right]=\left[1_{+} a_{-} \infty_{-}\right] \cdot\left[\infty_{-}\right]=\left[\infty_{-}\right] \cdot\left[1_{+} a_{+} \infty_{+}\right],
$$

the right-multiplication by $\left[\infty_{-}\right]$interchanges $\alpha, \beta$, and the left-multiplication by $\left[\infty_{-}\right]$interchanges the third and fourth arguments of $H l$, which is not the same 
TABLE 3. An isomorphism between $G_{\text {asym }, 0}(4) \cong \mathcal{D}_{3}$, the transformation group of $H l$, and $S_{4}$, the symmetric group on 4 letters.

\begin{tabular}{|c|l|}
\hline \multicolumn{1}{|c|}{$g \in \mathcal{D}_{3}$} & $g^{\prime} \in S_{4}$ \\
\hline \hline$\left[1_{+}\right]\left[a_{+}\right]\left[\infty_{+}\right]$ & () \\
{$\left[1_{-}\right]\left[a_{+}\right]\left[\infty_{-}\right]$} & $(12)(34)$ \\
{$\left[1_{+}\right]\left[a_{-}\right]\left[\infty_{-}\right]$} & $(13)(24)$ \\
{$\left[1_{-}\right]\left[a_{-}\right]\left[\infty_{+}\right]$} & $(14)(23)$ \\
\hline$\left[1_{+} \infty_{+}\right]\left[a_{+}\right]$ & $(12)$ \\
{$\left[1_{-} \infty_{-}\right]\left[a_{+}\right]$} & $(34)$ \\
{$\left[1_{-} \infty_{+}\right]\left[a_{-}\right]$} & $(1423)$ \\
{$\left[1_{+} \infty_{-}\right]\left[a_{-}\right]$} & $(1324)$ \\
\hline$\left[1_{+} a_{+}\right]\left[\infty_{+}\right]$ & $(14)$ \\
{$\left[1_{+} a_{-}\right]\left[\infty_{-}\right]$} & $(1342)$ \\
{$\left[1_{-} a_{+}\right]\left[\infty_{-}\right]$} & $(1243)$ \\
{$\left[1_{-} a_{-}\right]\left[\infty_{+}\right]$} & $(23)$ \\
\hline
\end{tabular}

\begin{tabular}{|c|l|}
\hline \multicolumn{1}{|c|}{$g \in \mathcal{D}_{3}$} & \multicolumn{1}{|c|}{$g^{\prime} \in S_{4}$} \\
\hline \hline$\left[1_{+} a_{+} \infty_{+}\right]$ & $(142)$ \\
{$\left[1_{+} a_{-} \infty_{-}\right]$} & $(134)$ \\
{$\left[1_{-} a_{-} \infty_{+}\right]$} & $(123)$ \\
{$\left[1_{-} a_{+} \infty_{-}\right]$} & $(243)$ \\
\hline$\left[1_{+}\right]\left[a_{+} \infty_{+}\right]$ & $(24)$ \\
{$\left[1_{-}\right]\left[a_{-} \infty_{+}\right]$} & $(1234)$ \\
{$\left[1_{+}\right]\left[a_{-} \infty_{-}\right]$} & $(13)$ \\
{$\left[1_{-}\right]\left[a_{+} \infty_{-}\right]$} & $(1432)$ \\
\hline$\left[1_{+} \infty_{+} a_{+}\right]$ & $(124)$ \\
{$\left[1_{-} \infty_{+} a_{-}\right]$} & $(234)$ \\
{$\left[1_{-} \infty_{-} a_{+}\right]$} & $(143)$ \\
{$\left[1_{+} \infty_{-} a_{-}\right]$} & $(132)$ \\
\hline
\end{tabular}

thing. Regardless of how $\left[1_{+} a_{-} \infty_{+}\right]$is factored, it follows from Table 2 that the corresponding transformation of $\mathrm{Hl}$ is

$$
\begin{aligned}
& H l(a, q ; \alpha, \beta, \gamma, \delta ; x) \\
& \quad=\left(1-\frac{x}{a}\right)^{-\alpha} H l\left(\frac{1}{1-a}, \frac{q-\gamma \alpha}{a-1} ;-\beta+\gamma+\delta, \alpha, \gamma, \alpha-\beta+1 ; \frac{x}{x-a}\right) .
\end{aligned}
$$

(For example, this can be obtained from the transformation indexed by $\left[1_{+} a_{-} \infty_{-}\right] \in$ $\mathcal{D}_{3}$, which is listed in the table, by interchanging $\alpha, \beta$.) Since $\left[1_{+} a_{-} \infty_{+}\right]$generates a cyclic group of order 6 , iterating (5.8) will generate six transformations of $\mathrm{Hl}$, including the identity. That is, up to interchange of $\alpha, \beta$, it will generate exactly one-fourth of the 24 transformations of $\mathrm{Hl}$, i.e., one-fourth of the 24 equivalent expressions for the first Frobenius solution in Table 2.

To see the consequences of the transformation group of $\mathrm{Hl}$ being isomorphic to $S_{4}$, one should index the 24 equivalent expressions for it not by $g \in \mathcal{D}_{3}$, but by the corresponding element $g^{\prime} \in S_{4}$. An isomorphism from $\mathcal{D}_{3}$ to $S_{4}$ is not difficult to construct, starting with a correspondence between their Coxeter generators. Such an isomorphism appears in Table 3. with each $g^{\prime} \in S_{4}$, the symmetric group on $1,2,3,4$, given in cycle notation. Since $S_{4}$ has no outer automorphisms, any isomorphism from $\mathcal{D}_{3}$ to $S_{4}$ will be the same as this, up to renumbering of 1,2,3,4. This isomorphism has an elegant interpretation that can be traced back to Klein. The group $\mathcal{D}_{3}$ can be understood as acting on a cube, as its group of rotational symmetries. The cube's three principal axes, which pierce the centers of opposite sides, correspond to the singular points $1, a, \infty$. Under the action of any $g \in \mathcal{D}_{3}$ these axes are permuted, and possibly reversed, with reversal of an axis indicated by a negative sign in the annotated cycle representation of $g$. Since the action of $\mathcal{D}_{3}$ on the cube is faithfully represented by its action on the cube's four principal diagonals, which it can permute arbitrarily, the isomorphism $\mathcal{D}_{3} \cong S_{4}$ follows.

In this representation the Pfaff and twisted Pfaff transformations, $\left[1_{+} \infty_{+}\right]$and $\left[1_{-} \infty_{-}\right]$, appear as (12) and (34). Each is a $180^{\circ}$ rotation about an axis passing through the midpoints of two opposite edges of the cube. The Euler transformation $\left[1_{-}\right]\left[\infty_{-}\right]$appears as $(12)(34)$, making it obvious that it belongs with them in a Klein 4 -group. It is a $180^{\circ}$ rotation about the $a$-axis. This representation also makes it 
possible to visualize the extension of $\mathcal{D}_{3}$ to $\mathcal{B}_{3}=\mathcal{D}_{3} \rtimes_{\Gamma} \mathbb{Z}_{2}$. On $\mathcal{D}_{3}$ represented as $S_{4}$ in this way, the twisting action of the involution [ $\left.\infty_{-}\right]$, which generates the $\mathbb{Z}_{2}$ factor and interchanges the exponents at infinity, turns out to be conjugation by the permutation (14)(23).

The group $S_{4}$ is doubly generated. For example, it is generated by the involution (14) and the 4-cycle (1423), which by Table 3 correspond to $\left[1_{+} a_{+}\right]$ and $\left[1_{-} \infty_{+}\right]\left[a_{-}\right]$in $\mathcal{D}_{3}$. It follows that the full set of 24 transformations of $\mathrm{Hl}$ is generated by only two well-chosen transformations, such as (by Table 2)

$$
\begin{aligned}
& H l(a, q ; \alpha, \beta, \gamma, \delta ; x) \\
& =H l\left(\frac{1}{a}, \frac{q}{a} ; \alpha, \beta, \gamma, \alpha+\beta-\gamma-\delta+1 ; \frac{x}{a}\right) \\
& =(1-x)^{\beta-\gamma-\delta}\left(1-\frac{x}{a}\right)^{-\alpha-\beta+\gamma+\delta} H l\left(\frac{a}{a-1}, \frac{-q-\gamma[(\beta-\gamma-\delta) a-\alpha-\beta+\gamma+\delta]}{a-1}\right. \text {; } \\
& \left.-\beta+\gamma+1,-\beta+\gamma+\delta, \gamma, \alpha-\beta+1 ; \frac{x}{x-1}\right) .
\end{aligned}
$$

This is certainly not obvious, and would be difficult to prove without an understanding of the group structure of the set of transformations.

The preceding examples focused on the group-theoretic aspects of the 24 equivalent expressions for $\mathrm{Hl}$, the most important of the eight Frobenius solutions of the Heun equation. But the 24 expressions given in Table 2 for each of the remaining seven, which are bijective with the cosets of $\mathcal{D}_{3}$ in $\mathcal{D}_{4}$, may also prove useful in applications. This is because the two-point connection problem for the Heun equation has never been solved in full generality, unlike the corresponding problem for the hypergeometric equation [21]. No general formula expressing the pair of Frobenius solutions defined near a nonzero singular point (i.e., $x=1, a, \infty)$ as a combination of the pair defined near $x=0$, one of which is $H l$, has yet been found.

Some notes on Table 2, The 192 solutions were obtained with MAXIMA, the free version of the MACSYMA computer algebra system. The implementation of the algorithm outlined in Proposition 4.4 required approximately 300 lines of code.

The code functioned as follows. Each $g \in G_{\text {asym }}(4) \cong \mathcal{D}_{4}$ was generated as a pair $(h, \sigma) \in\left(\mathbb{Z}_{2}\right)^{3} \rtimes S_{4}$. Here $\sigma \in S_{4}$ determines the change of independent variable $x^{\prime}=P_{\sigma} x$, where $P_{\sigma}$ is one of the 24 Möbius transformations in the Heun group, listed in (3.2); and $h$ specifies the subsequent index tranformation, i.e., the negatings, if any, of exponent differences at the singular points $x^{\prime}=0,1, a^{\prime}$. The change of independent variable and the index transformation were applied in that order to the Heun equation (1.2), and the transformed parameter vector $\left(a^{\prime}, q^{\prime} ; \alpha^{\prime}, \beta^{\prime}, \gamma^{\prime}, \delta^{\prime}\right)$ was programmatically extracted from the transformed equation. The transformed accessory parameter $q^{\prime}$ was then re-expressed in as compact a form as possible. The simplification of $q^{\prime}$ sometimes required factoring quadratic polynomials in the exponent parameters. The first instance of this in the table occurs in the solution indexed by $\left[0_{-}\right]\left[1_{-} \infty_{+}\right]\left[a_{+}\right]$.

Each solution was then output in a linear (string) format, and converted to $\mathrm{HT}_{\mathrm{E}} \mathrm{X}$ format for inclusion in the table. Conversion and table construction were largely programmatic rather than manual. The standard tools sed and awk distributed with the GNU/Linux operating system were employed.

The chance of there being an error in Table 2 is very small. After the table was prepared in $\mathrm{A}_{\mathrm{E}} \mathrm{X}$ format, each of the 192 expressions in it was programmatically converted to the syntax of the $\mathrm{C}$ programming language, and a straightforward C program (a "driver") that evaluates the 24 expressions for any of the 8 distinct 
Frobenius solutions was written. In this program $H l$ was computed as the sum of a Heun series, the coefficients of which were calculated from the recurrence (5.4). The driver was executed with randomly chosen values for the Heun parameters $a, q, \alpha, \beta, \gamma, \delta$, and also for $x$ (always taken sufficiently near the appropriate singular point, of course). For any choice of these seven parameters, the 24 values of the specified Frobenius solution produced by the driver always agreed with one another. This provides strong evidence of the correctness of Table 2, in toto.

The 192 expressions of the table were compared with the 48 expressions published by Heun in his original paper [11, and those later published by Snow [25, chap. VII]. Heun's table includes every fourth line of Table 2, in order (the first, fifth, ninth, etc.). Sadly, almost all his solutions were found to be incorrect. It is not clear why he made so many errors, since it is only the transformed accessory parameter $q^{\prime}$, which he omitted from each solution, that poses severe difficulties in hand computation. It is quite feasible to compute the exponent parameters of each $H l$, and the prefactor if any, by hand, by manipulating Riemann P-symbols. He had especial problems with prefactors. For example, each of the final 12 solutions in his 48 -solution table has an erroneous prefactor (either $x^{\alpha}$ or $x^{\beta}$ ), despite being otherwise correct.

Snow used an individualistic notation in which the exponent parameters $\delta, \epsilon$ are interchanged, and the accessory parameter is the negated quantity $b=-q$. To facilitate comparison with his results, a version of Table 2 using his notation was prepared. (This alternative version is available on request from the author.) His 25 solutions, each of which includes the value of $b^{\prime}$, proved to be far more reliable than Heun's. No errors were found, and only a single misprint. In his formula VII(21c), which gives the solution listed as $\left[\infty_{+} 0_{+} 1_{+}\right]\left[a_{+}\right]$in Table 2 , the argument of his local Heun function should be $1 /(1-z)$ rather than $1 /(z-1)$.

The several solutions supplied by the late F. Arscott in his valuable review of the Heun equation [19, Part A] were also examined. In his $(2.2 .5, \mathrm{I})-(2.2 .5$, VIII) he gives the parameter vectors $\left(a^{\prime}, q^{\prime} ; \alpha^{\prime}, \beta^{\prime}, \gamma^{\prime}, \delta^{\prime}\right)$ of the eight solutions indexed by even-signed permutations of the form $\left[0_{ \pm}\right]\left[1_{ \pm}\right]\left[a_{ \pm}\right]\left[\infty_{ \pm}\right]$. His transformed parameters are almost entirely correct, but there is one misprint. The term " 1 " in the value for $q^{\prime}$ in $(2.2 .5, \mathrm{VI})$, which corresponds to the solution $\left[0_{-}\right]\left[1_{+}\right]\left[a_{-}\right]\left[\infty_{+}\right]$ in Table 2, should be "2". This misprint has already been noted [20. Also, sign errors were found in (3.4.9)-(3.4.10). Due to these, his formula (3.4.11), which is the Pfaff-transformed $\mathrm{Hl}$ indexed in Table 2 by $\left[0_{+}\right]\left[1_{+} \infty_{+}\right]\left[a_{+}\right]$, is unfortunately incorrect. It is missing its prefactor as well, but that surely counts as a misprint.

A few additional local solutions of the Heun equation, expressed in terms of $\mathrm{Hl}$, can be found scattered in the literature. For instance, Wakerling [27, §IV] derived the solution indexed by $\left[\infty_{+} 0_{+}\right]\left[1_{+}\right]\left[a_{+}\right]$by manually applying to the Heun equation the change of independent variable $x^{\prime}=1 / x$, etc. Her expression for the transformed accessory parameter is correct. Schmitz and Fleck, who were the first in recent times to call attention to the problems with Heun's original list, carefully worked out the solutions indexed by $\left[\infty_{+} 0_{+} 1_{+} a_{+}\right]$and $\left[\infty_{-} 0_{+} 1_{+} a_{-}\right]$for their paper [22, $\S 6.3 .1]$. It is painful to report that their Eq. (102), which gives the corresponding accessory parameters, contains misprints (missing exponents).

In the course of writing the present paper two further lists of local solutions of the Heun equation came to light, neither of which is cited in standard references. One is in the 1898 inaugural dissertation of Karl Franz [7, who derived 35 of the 192, though like Heun, he omitted accessory parameters. He used an idiosyncratic 
parametrization: in our (and Heun's) notation, his exponent parameters $\alpha, \beta, \gamma, \gamma_{1}$ equal $\alpha, \beta, \gamma+\epsilon, \gamma$. His 35 solutions appear to be correct, so far as they go.

The other list is in the unpublished Ph.D. dissertation of Shun-Teh Ma 14, which has languished in the Berkeley stacks for many decades. Though Ma evidently did not have a full group-theoretic understanding of the transformation theory of the Heun equation, he attempted to work out all 192 expressions for its Frobenius solutions, including the accessory parameters, by hand computation. This doubtless required prodigious labor on his part. Unfortunately, a careful comparison of his 192 expressions with Table 2 reveals that 64 have incorrect exponent parameters or prefactors, and 156 have incorrect accessory parameters. Of his 192 expressions, only 31 have neither sort of error.

A final note. The number of local solutions of the Heun equation of the form considered in this paper, viz., $\mathrm{Hl}$ with a projectively transformed argument, multiplied by powers of $x, x-1$, and $x-a$, has been variously given in the literature. Whittaker and Watson 28 give the usual figure of 192 , which can surely be traced back to Heun. But Ince [12, p. 394] gives a figure of 64, i.e., 192/3, and Arscott [19, Part A] gives a figure of 96 , i.e., 192/2. The variations evidently arise from differing interpretations of what it means for two expressions for the same Frobenius solution to be distinct. We have been unable to find a convincing explanation for Ince's figure, but Arscott explained the origin of his. The involutory Heun transformation corresponding to $\left[0_{+}\right]\left[1_{+} a_{+}\right]\left[\infty_{+}\right]$in Table 2 , i.e.,

$$
H l(a, q ; \alpha, \beta, \gamma, \delta ; x)=H l\left(\frac{1}{a}, \frac{q}{a} ; \alpha, \beta, \gamma, \alpha+\beta-\gamma-\delta+1 ; \frac{x}{a}\right),
$$

is unique in that its right-hand side includes no prefactor. Applying it to any of the 192 expressions of the table is quite easy. Doing so yields another such expression, so as Arscott says, the 192 are linked in pairs. In group-theoretic language, his 96 "distinct" solutions are indexed not by the elements of $\mathcal{D}_{4}$, but rather by the cosets in $\mathcal{D}_{4}$ of the two-element group generated by $\left[0_{+}\right]\left[1_{+} a_{+}\right]\left[\infty_{+}\right]$. Left and right cosets are different, in general, and he did not specify which pairing he preferred.

\section{REFERENCES}

[1] M. Abramowitz and I. A. Stegun, editors. Handbook of Mathematical Functions with Formulas, Graphs, and Mathematical Tables. Number 55 in Applied Mathematics Series. National Bureau of Standards, Washington, DC, 1964. MR0167642 (29:4914)

[2] G. E. Andrews, R. Askey, and R. Roy. Special Functions, volume 71 of Encyclopedia of Mathematics and its Applications. Cambridge University Press, Cambridge, UK, 1999. MR1688958 (2000g:33001)

[3] M. Baake. Structure and representations of the hyperoctahedral group. J. Math. Phys., 25(11):3171-3182, 1984. MR0761836 (85j:20008)

[4] F. Brenti. q-Eulerian polynomials arising from Coxeter groups. European J. Combin., 15(5):417-441, 1994. MR1292954 (95i:05013)

[5] B. Dwork. On Kummer's twenty-four solutions of the hypergeometric differential equation. Trans. Amer. Math. Soc., 285(2):497-521, 1984. MR0752488(86a:12002)

[6] A. Erdélyi, editor. Higher Transcendental Functions. McGraw-Hill, New York, 1953-55. Also known as The Bateman Manuscript Project.

[7] K. Franz. Untersuchungen über die lineare homogene Differentialgleichung 2. Ordnung der Fuchs'schen Klasse mit drei im Endlichen gelegenen singulären Stellen. Inaugural dissertation, Friedrichs-Universität Halle-Wittenberg, 1898.

[8] F. Gesztesy and R. Weikard. Treibich-Verdier potentials and the stationary (m)KdV hierarchy. Math. Z., 219(3):451-476, 1995. MR1339715(96e:14030) 
[9] J. J. Gray. Linear Differential Equations and Group Theory. Birkhäuser, Boston/Basel, 2nd edition, 2000. MR:1751835 (2000m:34002)

[10] L. C. Grove and C. T. Benson. Finite Reflection Groups. Springer-Verlag, New York/Berlin, 2nd edition, 1985. MR0777684 (85m:20001)

[11] K. Heun. Zur Theorie der Riemann'schen Functionen zweiter Ordnung mit vier Verzweigungspunkten. Math. Ann., 33:161-179, 1889.

[12] E. L. Ince. Ordinary Differential Equations. Dover, New York, 1944; reprint of the 1926 edition. MR0010757 (6:65f)

[13] E. E. Kummer. Über die hypergeometrische Reihe $1+\frac{\alpha \cdot \beta}{1 . \gamma} x+\frac{\alpha(\alpha+1) \beta(\beta+1)}{1.2 \cdot \gamma(\gamma+1)} x^{2}+$ $\frac{\alpha(\alpha+1)(\alpha+2) \beta(\beta+1)(\beta+2)}{1.23(\gamma+1)(\gamma+2)} x^{3}+\ldots$ J. Reine Angew. Math., 15:39-83, 127-172, 1836.

[14] S.-T. Ma. Relations Between the Solutions of a Linear Differential Equation of Second Order with Four Regular Singular Points. Ph.D. dissertation, University of California, Berkeley, Dept. of Mathematics, 1934.

[15] R. S. Maier. On reducing the Heun equation to the hypergeometric equation. J. Differential Equations, 213(1):171-203, 2005. MR2139342 (2006a:34245)

[16] S. V. Oblezin. Discrete symmetries of systems of isomonodromic deformations of second-order Fuchsian differential equations. Funct. Anal. Appl., 38(2):111-124, 2004. Russian original in Funktsional. Anal. i Prilozhen, 38(2):38-54, 2004. MR2086626 (2005g:32017)

[17] E. G. C. Poole. Linear Differential Equations. Oxford University Press, Oxford, 1936.

[18] R. T. Prosser. On the Kummer solutions of the hypergeometric equation. Amer. Math. Monthly, 101(6):535-543, 1994. MR 1274975 (95b:33005)

[19] A. Ronveaux, editor. Heun's Differential Equations. Oxford University Press, Oxford, 1995. MR1392976 (98a:33005)

[20] A. Ronveaux. Factorization of Heun's differential operator. Appl. Math. Comput., 141(1):177184, 2003. MR1986079 (2004d:34180)

[21] R. Schäfke and D. Schmidt. The connection problem for general linear ordinary differential equations at two regular singular points with applications to the theory of special functions. SIAM J. Math. Anal., 11(5):848-862, 1980. MR0586913 (82a:34010a)

[22] F. Schmitz and B. Fleck. On the propagation of linear 3-D hydrodynamic waves in plane non-isothermal atmospheres. Astron. Astrophys. Suppl. Ser., 106(1):129-139, 1994.

[23] A. O. Smirnov. Elliptic solitons and Heun's equation. In V. B. Kuznetsov, editor, The Kowalevski Property, no. 32 in CRM Proc. Lecture Notes, pages 287-305. American Mathematical Society, Providence, RI, 2002. MR.1916788 (2003f:34183)

[24] A. O. Smirnov. Finite-gap solutions of Fuchsian equations. Lett. Math. Phys. 76(2-3):297316, 2006. MR2238723

[25] C. Snow. Hypergeometric and Legendre Functions with Applications to Integral Equations of Potential Theory. Number 19 in Applied Mathematics Series. National Bureau of Standards, Washington, DC, 2nd edition, 1952. MR0048145(13:988b)

[26] V. S. Varadarajan. Linear meromorphic differential equations: A modern point of view. Bull. Amer. Math. Soc., 33(1):1-42, 1996. MR1339809(96h:34011)

[27] V. W. Wakerling. The relations between solutions of the differential equation of the second order with four regular singular points. Duke Math. J., 16:591-599, 1949. MR0035347 $(11: 720 \mathrm{e})$

[28] E. T. Whittaker and G. N. Watson. A Course of Modern Analysis. Cambridge University Press, Cambridge, UK, 1996, reprint of the fourth (1927) edition. MR1424469 (97k:01072)

[29] M. Yoshida. A presentation of the fundamental group of the configuration space of 5 points on the projective line. Kyushu J. Math., 48(2):283-289, 1994. MR1294531 (95f:57003)

Departments of Mathematics and Physics, University of Arizona, Tucson, Arizona 85721

E-mail address: rsm@math.arizona.edu

$U R L:$ http://www. math. arizona.edu/ ${ }^{\sim} \mathrm{rsm}$ 